\title{
On the comparison of LES data-driven reduced order approaches for hydroacoustic analysis
}

\author{
Mahmoud Gadalla*1, Marta Cianferra ${ }^{\dagger 2}$, Marco Tezzele ${ }^{\ddagger 1}$, Giovanni Stabile ${ }^{\S 1}$, \\ Andrea Mola ${ }^{\mathbb{1}}$, and Gianluigi Rozza ${ }^{\| 1}$ \\ ${ }^{1}$ Mathematics Area, mathLab, SISSA, via Bonomea 265, I-34136 Trieste, Italy \\ 2Industrial and Environmental Fluid Dynamic Research Group, University of \\ Trieste, Trieste, Italy
}

December 29, 2020

\begin{abstract}
In this work, Dynamic Mode Decomposition (DMD) and Proper Orthogonal Decomposition (POD) methodologies are applied to hydroacoustic dataset computed using Large Eddy Simulation (LES) coupled with Ffowcs Williams and Hawkings (FWH) analogy. First, a low-dimensional description of the flow fields is presented with modal decomposition analysis. Sensitivity towards the DMD and POD bases truncation rank is discussed, and extensive dataset is provided to demonstrate the ability of both algorithms to reconstruct the flow fields with all the spatial and temporal frequencies necessary to support accurate noise evaluation. Results show that while DMD is capable to capture finer coherent structures in the wake region for the same amount of employed modes, reconstructed flow fields using POD exhibit smaller magnitudes of global spatiotemporal errors compared with DMD counterparts. Second, a separate set of DMD and POD modes generated using half the snapshots is employed into two data-driven reduced models respectively, based on DMD mid cast and POD with Interpolation (PODI). In that regard, results confirm that the predictive character of both reduced approaches on the flow fields is sufficiently accurate, with a relative superiority of PODI results over DMD ones. This infers that, discrepancies induced due to interpolation errors in PODI is relatively low compared with errors induced by integration and linear regression operations in DMD, for the present setup. Finally, a post processing analysis on the evaluation of FWH acoustic signals utilizing reduced fluid dynamic fields as input demonstrates that both DMD and PODI data-driven reduced models are efficient and sufficiently accurate in predicting acoustic noises.
\end{abstract}

\section{Contents}

1 Introduction

2 Methodology 4

2.1 Full order model . . . . . . . . . . . . . . . . . . . . . . 4

2.2 Acoustic model . . . . . . . . . . . . . . . . . . . 5

2.3 Dynamic mode decomposition . . . . . . . . . . . . . . . . . 7

2.4 Proper orthogonal decomposition with interpolation . . . . . . . . . 8

*mahmoud.gadalla@sissa.it

†marta.cianferra@dia.units.it

$\ddagger$ marco.tezzele@sissa.it

\$giovanni.stabile@sissa.it

$\mathbb{I}_{\text {andrea.mola@sissa.it }}$

|| gianluigi.rozza@sissa.it 
3.1 Full order CFD . . . . . . . . . . . . . . . . . . . . 9

3.2 Modal decomposition . . . . . . . . . . . . . . . . . . . . 10

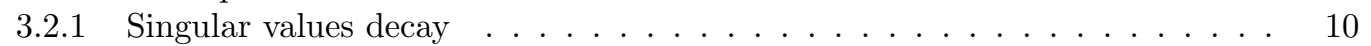

3.2.2 Modal representation . . . . . . . . . . . . . . . . . . . 10

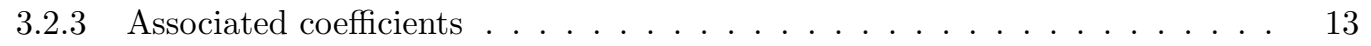

3.3 Flow fields reconstruction .................... 13

3.3.1 Global error . . . . . . . . . . . . . . . . . 15

3.3.2 Fields visualization . . . . . . . . . . . . . . . . . . . 15

3.3.3 Error statistics in the wake region . . . . . . . . . . . . . 16

3.4 Fields mid cast using DMD and PODI . . . . . . . . . . . . . . . . . . 18

3.4.1 Prediction error analysis . . . . . . . . . . . . . . . 19

3.4.2 Coherent structures . . . . . . . . . . . . . . . . . 20

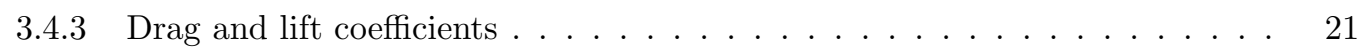

3.5 Spectral analysis . . . . . . . . . . . . . . . . . . . . . 22

3.5.1 Data probes inside and outside the wake region . . . . . . . . . . 22

3.5.2 Fast Fourier transform . . . . . . . . . . . . . . . . . 24

3.6 Acoustic analysis . . . . . . . . . . . . . . . . . . 26

4 Conclusions and perspectives 28

\begin{tabular}{ll} 
A ROMs cross-comparison & 29 \\
\hline
\end{tabular}

\section{Introduction}

In several engineering fields, there has been recently a growing need to include fluid dynamic performance evaluation criteria associated with acoustic emissions. This is, for example, the case in aircraft engine design, car manufacturing, and ship design optimization. A particular motivation behind the present research article is to investigate and propose a methodology that can be successively used for the noise level prediction of naval propellers since the early design process [16, 42].

The need for a reduction of the acoustic emissions through ship design optimization usually involves virtual prototyping and parametric high fidelity simulations. Thanks to the increase of the available computational resources, a deeper insight towards the complex physics associated with hydroacoustic phenomena has become nowadays affordable with unprecedented spatial and temporal scales (see, for example, wall-resolving Large Eddy Simulation (LES) 67, 48]). However, the enormous data sizes resulting from such simulations pose several challenges on the input/output operations, post-processing, or the long-term data storage. On the other hand, hybrid techniques such as, among others, Detached-Eddy Simulation or wall-layer model LES (WLES), have allowed obtaining eddy resolving field data with a reasonable use of computational resources. These techniques are, however, still expensive for an early stage design process, when a number of different geometric configurations has to be rapidly analysed to restrict the range of variation of the principal design parameters. Therefore, seeking a suitable data compression strategy that allows extracting the most relevant and revealing information in a reduced order manner, hence providing quick access as well as efficient data storage, becomes a crucial asset.

Through multidisciplinary scale, efforts have been made to realize optimal shape design for underwater noise sources, including ship hulls [13, 98, 93, and propellers 62, 99, using efficient geometrical parameterization techniques [95, 32. On the acoustic side, the development of new generation noise prediction tools was considered a major focus in this work. Particularly, based on the Ffowcs Williams and Hawkings (FWH) analogy 29, several improvements have been developed. For example, Cianferra et al. [15] compared several implementations of the non-linear quadrupole term, highlighting its significant contribution to the overall hydrodynamic noise emissions in wide range of frequencies. In a companion paper [14], they showed the effect of shape deformation on the radiated noise for elementary geometries. On a more engineering level, the generated hydrodynamic noise from a benchmark marine propeller was evaluated in open sea conditions [16, 17] using FWH coupled with LES.

The aforementioned hydroacoustic models are typically described by a system of non-linear Partial Differential Equations (PDEs), the resolution of which results in the fluid dynamic fields 
necessary to reproduce the noise source for an acoustic predictions. In fact, a reliable reconstruction of the noise source is crucially dependent on the flow structures and the resolved spatial and temporal scales of the fluid dynamic fields (for a discussion, see [8]).

Besides LES works [86, 2, 65, 48, 14, 17, 15, several fluid dynamic models have been employed in marine hydroacoustics. To name a few, we mention the potential flow theory [47, boundary element method [85, Reynolds Averaged Navier-Stokes equations (RANS) [36, 42, 1], DES [57, and Direct Numerical Simulation (DNS) [19, 84, 79. Being regarded as an optimal advance between RANS and DNS, recent literature has reported LES to be the most suitable model which reproduces the noise source with high level of realism [65, 2, 41]. The resolution of the described system of PDEs using standard discretization methods (finite elements, finite volumes, finite differences), which we will refer hereafter as the Full Order Model (FOM), allows for high fidelity acoustic evaluation.

Although hybrid fluid dynamic techniques constitute a good compromise between accuracy and computational cost for engineering purposes, they are still expensive in the case of parametric analysis and shape optimization. To overcome this issue, the development of a Reduced Order Model (ROM) [77, 174, 39, 73] — which alleviates both the computational complexity and data capacity - becomes essential. Moreover, several ROM developments have been realized to account for various effects including subgrid scales [40], heat transfer [34] and stabilization of the Galerkin projection 89 .

One of the necessary assumptions to construct an efficient ROM is that, the solution manifold of the underlying problem lies in a low dimensional space and, therefore, can be expressed in terms of a linear combination of a few number of global basis functions (reduced basis functions). Among various techniques to generate such reduced basis set, the Proper Orthogonal Decomposition (POD) and the Dynamic Mode Decomposition (DMD) have been widely exploited due to their versatile properties [92. POD provides a set of orthogonal and optimal basis functions [49], whereas DMD computes a set of modes with an intrinsic temporal behavior, hence it is particularly suited for time advancing problems 80. One of the main goals of this work is to understand whether these two established techniques are able to generate global basis functions which include all the wide range of frequencies associated with hydroacoustic phenomena, and therefore allow for accurate noise predictions. In addition, the interest is in assessing the efficiency of the modal decompositions algorithms, evaluating the amount of modal shapes required to accurately reproduce all high frequencies relevant to acoustic analysis. This investigation is of course a first, fundamental step towards developing efficient reduced order models for hydroacoustic applications. As will be thoroughly discussed, in the present work we only considered data-driven ROMs, but the POD and DMD efficiency assessment reported provides valuable information also for the development of projection based ROMs.

In literature, POD has been widely used for the past few decades to identify the coherent structures of turbulent flows (see, among others, 88, 3), and has been applied towards various flow conditions [59, 71. Correspondingly, DMD has been also exploited [80, 83. An intuitive question may arise considering the comparative performance. In that regard, several works have carried out both DMD and POD on various flow configurations. For instance, Liu et al. [103. conducted comprehensive analysis, concluding that DMD has the ability to clearly separate the flow coherent structure in both spatial and spectral senses, whereas POD was contaminated by other uncorrelated structures. Consistent to the previous, in [4 it was noted that DMD is useful when the main interest is to capture the dominant frequency of the phenomenon, while the optimality of the POD modes prevails for coherent structure identification that are energetically ranked. In highspeed train DES, Muld et al. 63. examined the convergence and reported that the most dominant DMD mode requires a longer sample time to converge when compared to the POD counterpart.

As discussed earlier, parametric studies such as shape optimization can result in extremely high computational costs. Possible ways to circumvent such issue could be degrading the high fidelity model, or restricting the design parameter space sampling. Since the only analysed parameter in the unsteady fluid dynamic problem is the time, in this work we decided to rely on datadriven ROMs. A significant source of error in data-driven ROMs is in fact associated with the interpolation of modal coefficients based on the problem parameters. Thus, such methods are an extremely valid alternative in presence of a one dimensional parameter space, in which interpolation errors are rather modest. In particular, POD with Interpolation (noted as PODI here-after) can be an adequate solution in such scenarios [54]. The basic idea is to exploit POD on selected ensemble of high fidelity solutions in the design space to identify the set of optimal basis functions and associated projection coefficients representing the solution dynamics. Such finite set of scalar 
coefficients are then utilized to train a response surface that allows predictions at parameter values that are not in the original high-fidelity ensemble. It was demonstrated that PODI can be efficiently utilized in various events: 1) enhancing the temporal resolution of experimental measurements [7, 2) optimal control [54, 3) reconstruction of incomplete data [27], 4) multi-dimensional parametric analysis [102, 30, 5) inverse design [11, 6) variable fidelity models [60, and more.

Both DMD and PODI are regarded as data-driven reduced models [10] since they operate on the snapshots produced from the FOM, and predict the system dynamics in a non-intrusive manner. In shape optimization context, DMD and PODI have been successively applied as in [20, 93, 21, 22, 96, 31] in naval engineering, 78, 26, 37] in automotive engineering, or [70, 43, in aeronautics. For acoustic analysis, only very few studies have been reported in literature. This can possibly include the DMD application as in [9, 44] or POD as in [35, 55, 87.

As previously demonstrated, there are very limited examples of ROMs specifically tailored for acoustic analysis. To the best of authors' knowledge, the literature is presently devoid of documents which characterize, in a thorough and systematic fashion, the performance of DMD and POD/I techniques on the hydroacoustic flow fields reconstruction or prediction.

The overarching goal of this study is to investigate the use of DMD and PODI on a hydroacoustic dataset corresponding to turbulent incompressible flow past sphere at $\mathrm{Re}=5000$, and computed using wall-resolving LES for the fluid dynamic fields, and FWH analogy with direct integration of the nonlinear quadrupole terms for the acoustic fields. In particular, the objectives of this work are 1) to understand the effect of DMD/POD modal truncation on the local and global reconstruction accuracy of the fluid dynamic fields, 2) to compare the efficiency of DMD and PODI on recovering the flow and spectral information when half the dataset are utilized, 3) to evaluate the performance of DMD and PODI in terms of data compression and dipole/quadrupole acoustic prediction.

The present article is organized as follows: first, a brief overview on the FOM (LES, FWH) and ROM (DMD, PODI) formulations and respective specific works are presented in section 2 .

In subsection 3.1. the FOM results are presented and then followed by modal analysis with DMD and POD in subsection 3.2. The reconstructed and predicted fluid dynamic data obtained from both ROMs are discussed in sections 3.3 and 3.4. while their spectral and acoustic performances are addressed in section 3.5 . Conclusions are drawn in section 4.

\section{Methodology}

First, the high fidelity data are generated and uniformly sampled in time using high fidelity simulations with LES turbulence modelling. The resulting matrix of snapshots is then factorized using Singular Value Decomposition (SVD) which is then used to construct both the DMD and POD spaces. The scalar coefficients resulting from the projection of the FOM data onto the POD space are used to train a continuous representation of the system temporal dynamics, i.e. PODI approach. In this context, both DMD and PODI are considered as linear approximation of the dynamical system of the snapshot matrix and, therefore, are used to predict the data at intermediate timesteps. The predicted snapshots are finally exploited to run a post-processing acoustic analogy and validate the accuracy of the noise generation compared to the FOM data. A summary of the procedure is presented in the flow chart in Figure 1. This section is organized as follows: in 2.1 the FOM is introduced recalling also the utilized LES turbulence model, in 2.2 the acoustic model used to perform the hydro-acoustic analysis is introduced, in 2.3 and 2.4 the non-intrusive pipeline used to perform model order reduction using DMD and PODI, respectively, is described.

\section{$2.1 \quad$ Full order model}

The full order model, adopted to provide the snapshots dataset as input for the reduced order model, is a Large Eddy Simulation. In LES, the large anisotropic and energy-carrying scales of motion are directly resolved through an unsteady three dimensional (3D) simulation, whereas the more isotropic and dissipative small scales of motion are confined in the sub-grid space. Scale separation is carried out through a filtering operation of the flow variables. In literature, the contribution of the Sub-Grid Scales (SGS) of motion on noise generation and propagation has been found negligible [66, 86. This means that the LES model can be considered accurate enough to provide a noise-source flow field, when compared to DNS. At the same time, the unsteady vortex and coherent structures can be of extreme importance when computing the noise signature. 


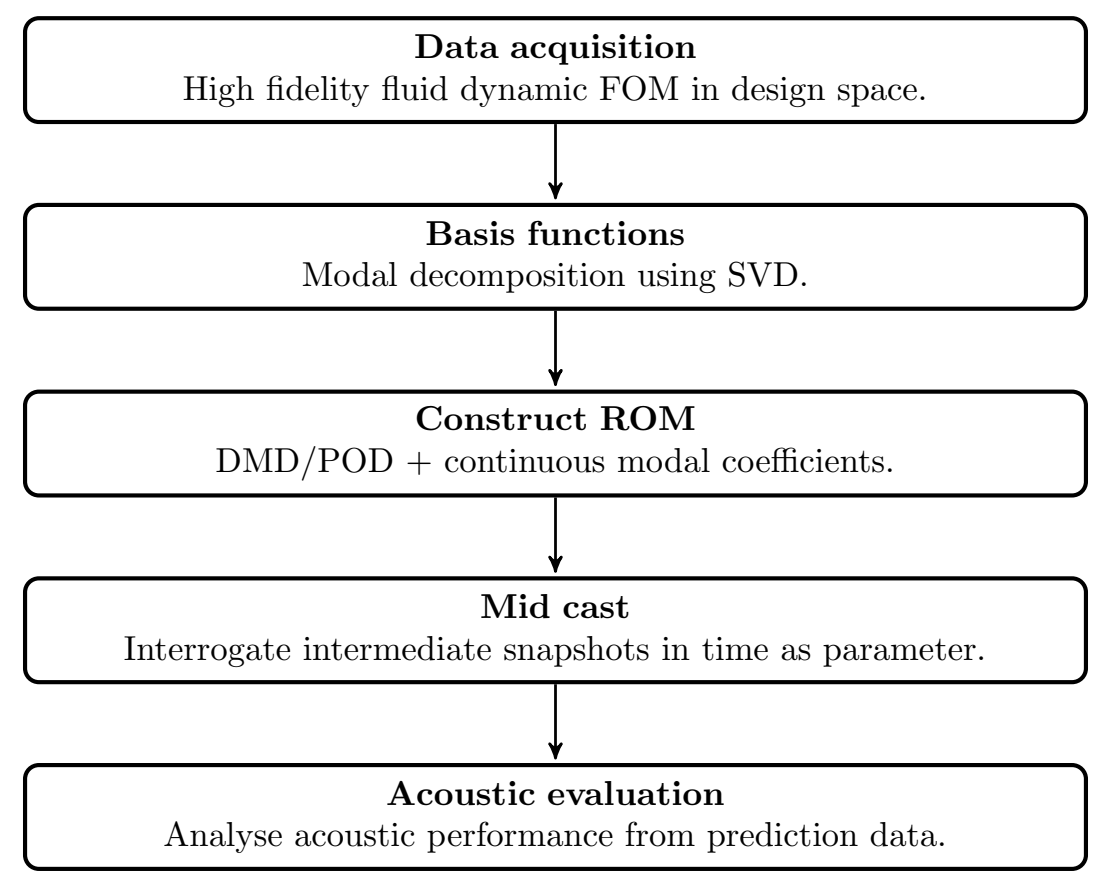

Figure 1: Flow chart of the FOM/ROM operations performed in the present study

Indeed, it has been shown (see among others [42]) that, in case of complex configurations (e.g. marine propellers), the RANS methodology may be unsatisfactory in reproducing the flow fields, adopted as input for the acoustic analysis.

The detailed numerical simulation of the flow around sphere was described in a previous work 14, where three different bluff bodies (a sphere, a cube and a prolate spheroid) were investigated concerning their noise signature. In the mentioned work, validations of the LES solver against experimental and DNS data were presented. In particular, the flow fields resulting from the employed framework reproduce the thin boundary layer developing along the wall-normal direction, the leading edge, and in the wake. Moreover, they were able to reproduce distributions of the friction coefficients, as well as capturing the underlying flow dynamics with a wide coverage of the spectral content.

The filtered Navier-Stokes equations in the incompressible regime are considered. Within this setting, the SGS stress tensor $\tau_{i j}^{\mathrm{sgs}}=\overline{u_{i} u_{j}}-\bar{u}_{i} \bar{u}_{j}$, which represents the effect of the unresolved fluctuations on the resolved motion, is modeled considering the Smagorinsky eddy-viscosity closure:

$$
\tau_{i j}^{\mathrm{sgs}}-\frac{1}{3} \tau_{k k}^{\mathrm{sgs}} \delta_{i j}=-2 \nu_{t} \bar{S}_{i j}, \text { with } \bar{S}_{i j}=\frac{1}{2}\left(\frac{\partial \bar{u}_{j}}{\partial x_{i}}+\frac{\partial \bar{u}_{i}}{\partial x_{j}}\right),
$$

and $\delta_{i j}$ is the isotropic second order tensor, while the overbar denotes the filtering operator. The SGS eddy viscosity $\nu_{t}$ is expressed as:

$$
\nu_{t}=\left(C_{s} \Delta\right)^{2}\left|\bar{S}_{i j}\right|,
$$

where $\Delta=\sqrt[3]{V_{c}}$ is the filter width which is defined as the cubic root of the cell volume $V_{c}$, and the Smagorinsky constant $C_{s}$ is computed dynamically using the Lagrangian procedure of [58], averaging over the fluid-particle Lagrangian trajectories.

\subsection{Acoustic model}

The acoustic model herein considered is the one proposed by Ffowcs Williams and Hawings [29], which is an extension of the Lighthill theory.

The basic idea behind the acoustic analogies is that pressure perturbation originates in the flow field and propagates in the far-field where the medium is assumed quiescent and uniform. The integral solution of the acoustic wave equation presented by Ffowcs Williams and Hawkings consists of surface and volume integrals, meaning that the sources of fluid-dynamic noise can be 
found as pressure-velocity fluctuations developing in the fluid region or as reflected pressure on an immersed solid surface.

We consider the original formulation presented in [29], and modified according to the works of Najafi et al. 64 and Cianferra et al. 14]. The modification of the original FWH equation takes into account the advection of acoustic waves. To account for the surrounding fluid moving at a constant speed (along the $x$ axis), the advective form of the Green's function must be considered. A derivation of the advective FWH equation is reported in 64, where the authors developed an integral solving formulation for the linear (surface) terms. The advective formulation of the volume term for the particular case of the wind tunnel flow is reported in [14].

In the present work, as done for the fluid dynamic part, we describe the formulation without dwelling into details, for which we refer to the previous works [14, 15.

The acoustic pressure $\hat{p}$, at any point $\mathbf{x}$ and time $t$, is represented by the sum of surface $\left(\hat{p}_{2 \mathrm{D}}\right)$ and volume $\left(\hat{p}_{3 \mathrm{D}}\right)$ integrals, respectively:

$$
\begin{aligned}
& 4 \pi \hat{p}_{2 \mathrm{D}}(\mathbf{x}, t)=\frac{1}{c_{0}} \frac{\partial}{\partial t} \int_{S}\left[\frac{\tilde{p} \hat{n}_{i} \hat{r}_{i}}{r^{*}}\right]_{\tau} d S+\int_{S}\left[\frac{\tilde{p} \hat{n}_{i} \hat{r}_{i}^{*}}{r^{* 2}}\right]_{\tau} d S \\
& 4 \pi \hat{p}_{3 \mathrm{D}}(\mathbf{x}, t)=\frac{1}{c_{0}^{2}} \frac{\partial^{2}}{\partial t^{2}} \int_{f>0}\left\{T_{i j}\left[\frac{\hat{r}_{i} \hat{r}_{j}}{r^{*}}\right]\right\}_{\tau} d V \\
& +\frac{1}{c_{0}} \frac{\partial}{\partial t} \int_{f>0}\left\{T_{i j}\left[\frac{2 \hat{r}_{i} \hat{r}_{j}^{*}}{r^{* 2}}+\frac{\hat{r}_{i}^{*} \hat{r}_{j}^{*}-R_{i j}^{*}}{\beta^{2} r^{* 2}}\right]\right\}_{\tau} d V \\
& +\int_{f>0}\left\{T_{i j}\left[\frac{3 \hat{r}_{i}^{*} \hat{r}_{j}^{*}-R_{i j}^{*}}{r^{* 3}}\right]\right\}_{\tau} d V \text {. }
\end{aligned}
$$

The pressure perturbation with respect to the reference value $p_{0}$ is denoted with $\tilde{p}=p-p_{0}, \hat{n}$ is the (outward) unit normal vector to the surface element $d S$, and $c_{0}$ is the sound speed. $\hat{r}$ and $\hat{r}^{*}$ are unit radiation vectors, $r$ and $r^{*}$ are the module of the radiation vectors $\mathbf{r}$ and $\mathbf{r}^{*}$ respectively. Their description is given in detail in [14.

Equation (4) contains two second-order tensors: $R_{i j}^{*}$ and the Lighthill stress tensor $T_{i j}$, the latter characterizing the FWH quadrupole term. Under the assumption of negligible viscous effects and iso-entropic transformations for the fluid in the acoustic field, the Lighthill tensor reads as:

$$
T_{i j}=\rho_{0} u_{i} u_{j}+\left(\tilde{p}-c_{0}^{2} \tilde{\rho}\right) \delta_{i j}
$$

where $\tilde{\rho}$ is the density perturbation of the flow which, in our case, is equal to zero. The surface integrals in Equation (3) are referred to as linear terms of the FWH equation and represent the loading noise term. The volume integrals in Equation (4) are slightly different from the standard FWH (non-advective) equation. For their derivation we consider a uniform flow with velocity $U_{0}$ along the streamwise direction.

Obviously, the direct integration of the volume terms gives accurate results, however, this method can be used if the calculation of the time delays can be omitted, otherwise the computational burden makes it unfeasible. In fact, the calculation of the time delays requires storing at each time step the pressure and velocity data related to the entire (noise-source) volume, in order to perform an interpolation over all the data.

However, for the case herein investigated, the evaluation of the non-dimensional Maximum Frequency Parameter (MFP) [15] (which is greater than unity for every microphone considered) allows to adopt the assumption of compact noise source. This means that, in the investigated case, the time delay is very small and the composition of the signals is not expected to contribute to the radiated noise. Since the evaluation of the time delays may be reasonably omitted, a remarkable saving of the CPU time is achieved, and the direct computation of the quadrupole volume terms becomes feasible.

In the two previous works 14, 15, as to perform a validation test for the acoustic model, the solution of the advective FWH equation was compared with the pressure signal provided by LES, considered as reference data. This comparison is useful to verify the ability of the acoustic post processing to accurately reconstruct the pressure field. Also, it points out the frequency range which is important to consider. 


\subsection{Dynamic mode decomposition}

Dynamic Mode Decomposition is a data-driven modal decomposition technique for analysing the dynamics of nonlinear systems [81, 82. DMD was developed in [80], and since then it has attracted a lot of attention and lead to diverse applications. Its popularity is also due to its equation-free nature since it relies only on snapshots of the state of the system at given times. DMD is able to identify spatiotemporal coherent structures, that evolve linearly in time, in order to approximate nonlinear time-dependent systems. For a comprehensive overview we refer to [50].

In the last years, many variants of the classical algorithm arose such as, for instance, the multiresolution DMD [51, DMD with control 68], and compressed DMD [28]. Higher order DMD 52 was developed for the cases that show limited spatial complexity but a very large number of involved frequencies. Sparsity promoting DMD to choose dynamically important DMD modes, can be found in [45. Kernel DMD was proposed in 101, where a large number of observables were able to be incorporated into DMD, exploiting the kernel trick. Randomized DMD for non-intrusive ROMs can be found in 5, 6. We also cite a new paradigm for data-driven modelling that uses deep learning and DMD for signal-noise decomposition [75. For numerical non-intrusive pipelines in applied sciences and industrial application we mention [74, 94, 97. From a practical point of view, the Python package PyDMD [24] contains all the major and most used aforementioned versions stemmed from the classical DMD in a user friendly way, from which the present work also exploits.

Here we present a brief overview of the standard algorithm, suppose a given discrete dataset $\boldsymbol{x}_{k} \in \mathbb{R}^{n}$, where $k \in[1, \ldots, m]$ denotes the snapshot number, and $n$ denotes the number of degrees of freedom. The $m$ snapshots can be arranged by columns into the data matrix $\boldsymbol{S}=$ $\left[\boldsymbol{x}_{1}, \boldsymbol{x}_{2}, \ldots, \boldsymbol{x}_{m-1}\right]$ and its temporal evolution $\dot{\boldsymbol{S}}=\left[\boldsymbol{x}_{2}, \boldsymbol{x}_{3}, \ldots, \boldsymbol{x}_{m}\right]$. Now, the objective is to seek a Koopman-like operator $[72] \boldsymbol{A}$, which is a best fit linear approximation that minimizes $\|\dot{\boldsymbol{S}}-\boldsymbol{A} \boldsymbol{S}\|_{F}$. Such operator is given as $\boldsymbol{A}=\dot{\boldsymbol{S}} \boldsymbol{S}^{\dagger} \in \mathbb{R}^{n \times n}$, where ${ }^{\dagger}$ is the Moore-Penrose pseudoinverse.

For practical reasons, the matrix $\boldsymbol{A}$ is not solved explicitly since it contains $n^{2}$ elements, from which $n$ is typically of several order of magnitudes, and is usually much larger than the number of snapshots $m$. Instead, a reduced operator $\widetilde{\boldsymbol{A}}$ is considered which has much lower dimension, yet it preserves the spectral information of $\boldsymbol{A}$. The procedure is defined in the following. First, a singular value decomposition (SVD) is performed on the snapshots matrix,

$$
\boldsymbol{S}=\boldsymbol{U} \boldsymbol{\Sigma} \boldsymbol{V}^{*} \approx \boldsymbol{U}_{r} \boldsymbol{\Sigma}_{r} \mathbf{V}_{r}^{*}
$$

where $r \leq(m-1)$ defines the SVD truncation rank, $\boldsymbol{U}_{r}$ is the truncated POD modes as it will be further discussed in the following section, and the three terms $\boldsymbol{U}_{r} \in \mathbb{C}^{n \times r}, \boldsymbol{\Sigma}_{r} \in \mathbb{C}^{r \times r}$ and $\boldsymbol{V}_{r}^{*} \in \mathbb{C}^{r \times(m-1)}$ denote the truncated SVD components. Second, the reduced operator $\widetilde{\boldsymbol{A}}$ is obtained through an $r \times r$ projection of $\boldsymbol{A}$ onto the POD modes $\boldsymbol{U}_{r}$, that is

$$
\widetilde{\boldsymbol{A}}=\boldsymbol{U}_{r}^{*} \boldsymbol{A} \boldsymbol{U}_{r}=\boldsymbol{U}_{r}^{*} \dot{\boldsymbol{S}} \boldsymbol{V}_{r} \boldsymbol{\Sigma}_{r}^{-1} \in \mathbb{C}^{r \times r} .
$$

The eigenspace of $\boldsymbol{A}$ is denoted as $\boldsymbol{\Phi}$ (also called the DMD modes), and it can be revealed through the low-rank projection of the eigenvectors $\boldsymbol{W}$ of $\widetilde{\boldsymbol{A}}$ onto the POD modes,

$$
\boldsymbol{\Phi}=\boldsymbol{U}_{r} \boldsymbol{W}
$$

where $\boldsymbol{W}$ is obtained from the eigendecomposition of $\widetilde{\boldsymbol{A}}$, i.e.

$$
\widetilde{A} \boldsymbol{W}=W \Lambda,
$$

and $\boldsymbol{\Lambda}$ is the diagonal matrix of eigenvalues of $\widetilde{\boldsymbol{A}}$ which is also considered the same matrix obtained from the eigendecomposition of $\boldsymbol{A}$. Finally, the DMD modes, $\boldsymbol{\Phi}$, and the diagonal matrix of eigenvalues, $\boldsymbol{\Lambda}$, are both used to provide a linear approximation to the solution vector $\boldsymbol{x}(t)$ at any time instance, that is

$$
\boldsymbol{x}(t) \approx \boldsymbol{\Phi} \exp (\boldsymbol{\Lambda} t) \boldsymbol{\Phi}^{\dagger} \boldsymbol{x}(0)
$$

In this work, we are going to characterize the DMD modes for velocity and pressure fields, and exploit them to make predictions between time instants. The reconstructed fields will be used to approximate the hydroacoustic noise. 


\subsection{Proper orthogonal decomposition with interpolation}

Proper orthogonal decomposition is a linear dimensionality reduction technique that is widely used to identify the underlying structures within large datasets. In general, a linear dimensionality reduction assumes that each snapshot in the dataset can be generated as a linear combination of a properly chosen small set of basis functions. In this regards, similar to the previous section, we introduce the snapshots matrix $\mathbf{S} \in \mathbb{R}^{n \times m}$ which now contains $m$ snapshots, thus $\mathbf{S}=\left[\mathbf{x}_{1} \mathbf{x}_{2} \cdots \mathbf{x}_{m}\right]$. Then, we assume it exists the linear combination $\mathbf{S} \approx \mathbf{U}_{r} \mathbf{C}$, where the matrix $\mathbf{U}_{r} \in \mathbb{R}^{n \times r}$ contains the vectors defining the basis functions, $r \leq m$, and $\mathbf{C} \in \mathbb{R}^{r \times m}$ is the matrix containing the linear coefficients. This approximation is equivalent to low-rank matrix approximation [56.

If we choose the basis functions that minimize the sum of the squares of the residual $\left(\mathbf{S}-\mathbf{U}_{r} \mathbf{C}\right)$, in the least square sense, then we recover the Proper Orthogonal Decomposition. In such case, the matrix $\mathbf{U}_{r}$ contains the so-called POD modes, and they can be recovered with the SVD of the snapshots matrix, as defined in Equation 6, for a low-rank truncation that retain the first $r$ modes. In fact, the error introduced by the truncation can measured as 69]:

$$
\begin{aligned}
\left\|\mathbf{S}-\mathbf{U}_{r} \boldsymbol{\Sigma}_{r} \mathbf{V}_{r}^{*}\right\|_{2}^{2} & =\lambda_{r+1}^{2}, \\
\left\|\mathbf{S}-\mathbf{U}_{r} \boldsymbol{\Sigma}_{r} \mathbf{V}_{r}^{*}\right\|_{F} & =\sqrt{\sum_{i=r+1}^{m} \lambda_{i}^{2}},
\end{aligned}
$$

where the subscripts 2 and $F$ refer to the Euclidean norm and to the Frobenius norm respectively, and $\lambda_{i}$ are the singular values arranged in descending order.

To approximate the solution manifold, after the projection of the original snapshots onto the POD space spanned by the POD modes, we interpolate the modal coefficients for new input parameters.

This non-intrusive data-driven approach is called POD with interpolation [54, 11, 12. A coupling with isogeometric analysis can be found in 33. For an enhancement of the method using active subspaces property, in the case of small computational budget devoted to the offline phase, see [25]. An open source Python implementation of PODI can be found in the EZyRB package [23]. For an implementation based on OpenFOAM [100] see the freely available ITHACA-FV library [91] from which the present work exploits.

The PODI method can be decomposed in two distinct phases: an offline phase, and an online one. In the first phase, given the solutions snapshots for given parameters $\mu_{k}$, we compute the POD modes $\phi_{k}$ (which construct the matrix $\mathbf{U}$ ) through SVD, and the corresponding modal coefficients for all the original snapshot with a projection. This results in

$$
\mathbf{x}_{k}=\sum_{j=1}^{m} \alpha_{j k} \phi_{j} \approx \sum_{j=1}^{r} \alpha_{j k} \phi_{j}, \quad \forall k \in[1,2, \ldots, m],
$$

where $\alpha_{j k}$ are the elements of the coefficient matrix $\mathbf{C}$.

After performing the offline phase, the $r$ most energetic modes are now obtained, and the corresponding modal coefficients $\alpha_{k}$ are used to construct the reduced space. Using the pairs $\left(\mu_{k}, \alpha_{k}\right)$ we are able to reconstruct the function that maps the input parameters to the modal coefficients. This function is interpolatory for the snapshots used in the training phase, hence the name $P O D$ with interpolation. With this surrogate function, we are able to reconstruct in real-time the solution fields of interest for an arbitrary parameter in the so-called online phase. In the present study, the input parameter is the time while the output fields are the velocity and pressure. Also, a cubic spline interpolation is considered for the modal coefficients. The degree of the interpolator was rather a modelling choice, given the flexibility provided by the simple, one-dimensional coefficient samples. In addition, the fairly easy implementation allowed for a robust performance that is comparative to simpler models, while smoother time response is herein preserved due to the higher order polynomial interpolation. Indeed, a more complicated application involving higher dimensional parameter space would require a careful choice of the hypothesis space, and typically more advanced machine learning (or deep learning) techniques would be more relevant, which is not the case in the present study.

In literature, several works have been devoted to study the accuracy of the projection coefficients manifold construction and performance. In 61 they explored the full factorial and latin hyper-cube 


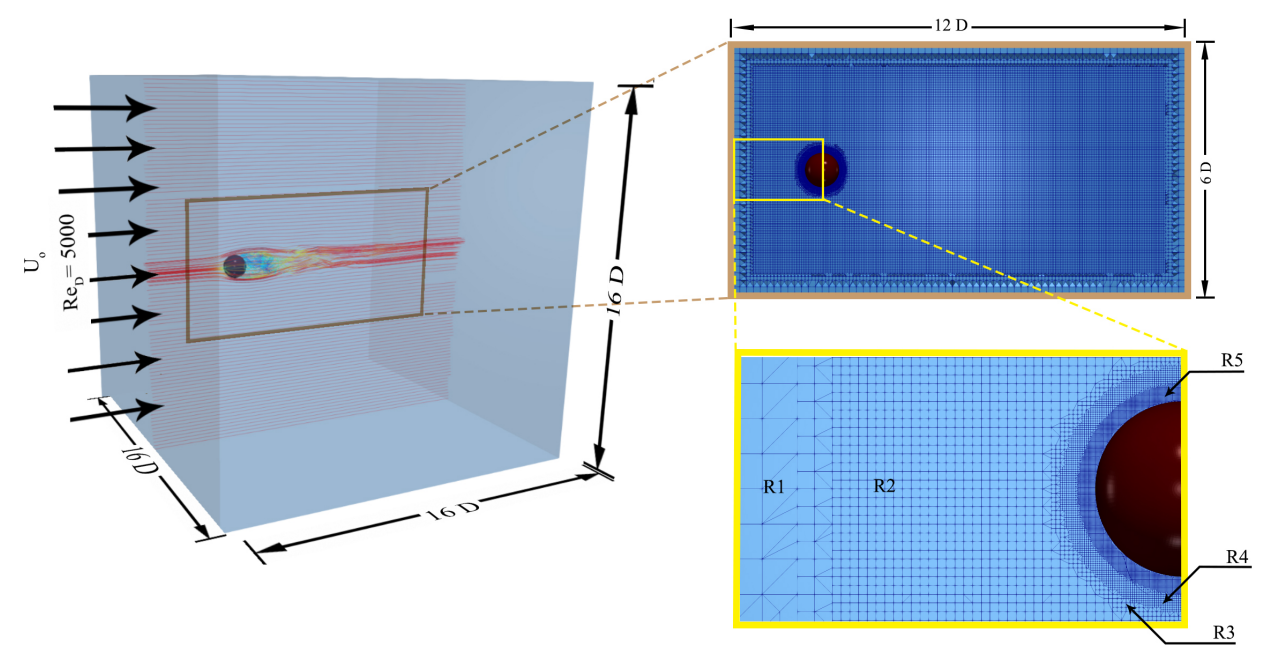

Figure 2: Sketch of the computational domain used for the FOM. The sphere diameter is $D=$ $0.01 \mathrm{~m}$. Successive mesh refinement layers $(R 2, R 3, R 4)$ are performed through cell splitting approach until reaching the finest grid spacing $0.001 D$ in the region $R 5$.

sampling techniques of the parameter space. The manifold representation was also studied by 46 , 61 mainly comparing between linear regression, polynomial, spline, finite difference, and radial basis functions (RBF). The high-order singular value decomposition (HOSVD) was demonstrated in [53] as an efficient method for flows with strong discontinuities. Kriging interpolation was utilized in [30] for $n$-dimensional approximation of potentially complex surfaces.

\section{Numerical results}

\subsection{Full order CFD}

The fluid dynamic fields are solved in the framework of the OpenFOAM library [100] which is based on the Finite Volume Method (FVM). The filtered Navier-Stokes equations are solved using the PISO pressure-velocity coupling algorithm implemented in the pisoFoam solver. The spatial derivatives are discretized through second-order central differences. Implicit time advancement runs according to the Euler scheme. The numerical algorithm, including the SGS closure, has been customized at the laboratory of Industrial and Environmental Fluid Mechanics (IE-Fluids) of the University of Trieste, and more details can be found in 18 .

The fluid dynamic full order model simulates a sphere of diameter $D=0.01 \mathrm{~m}$, immersed in a water stream with constant streamwise velocity $U_{0}=1 \mathrm{~m} / \mathrm{s}$. The kinematic viscosity is $\nu=2.0 \times 10^{-6} \mathrm{~m}^{2} / \mathrm{s}$, so that the Reynolds number based on the sphere diameter is $\operatorname{Re}_{D}=5000$.

The computational domain, depicted in Figure 2, is a box with dimensions $16 D \times 16 D \times 16 D$ along the $x, y$ and $z$ axes respectively. The sphere is located such that a distance of $12 D$ is attained downstream, along the $x$-axis, while it is centered with respect to the other axes. A zero-gradient condition is set for the pressure at the domain boundaries, except for the outlet where pressure is set to zero. The velocity is set to $U_{0}$ at the inlet, stress-free condition is set at the lateral boundaries, and zero-gradient condition is set for the velocity components at the outlet.

The grid, unstructured, and body-fitted, consists of about 5 millions of cells. It is created using the OpenFOAM snappyHexMesh utility. The grid spacing normal to the wall for the densest layer of cells (indicated as R5) is such to have first cell center within a wall unit $y^{+}\left(y^{+}=u_{\tau} y / \nu\right.$ with $u_{\tau}=\sqrt{\tau_{w} / \rho_{0}}$ and $\tau_{w}$ the mean shear stress). An $A$ posteriori analysis showed that about 5 grid points are placed within 10 wall units off the wall. The grid spacing is obtained through successive transition refinements (indicated as $R 3$ and $R 4$ in Figure 2). A refinement box around the body (named $R 2$ in Figure 2 ) is considered so as to obtain, in the wake region, a grid size of less than $0.1 D$ at a distance of $8 D$. Out of the region of interest, a coarser grid (indicated as $R 1$ ) allows for possible extension of the domain dimensions, and reducing possible disturbance effects coming from the boundaries. 


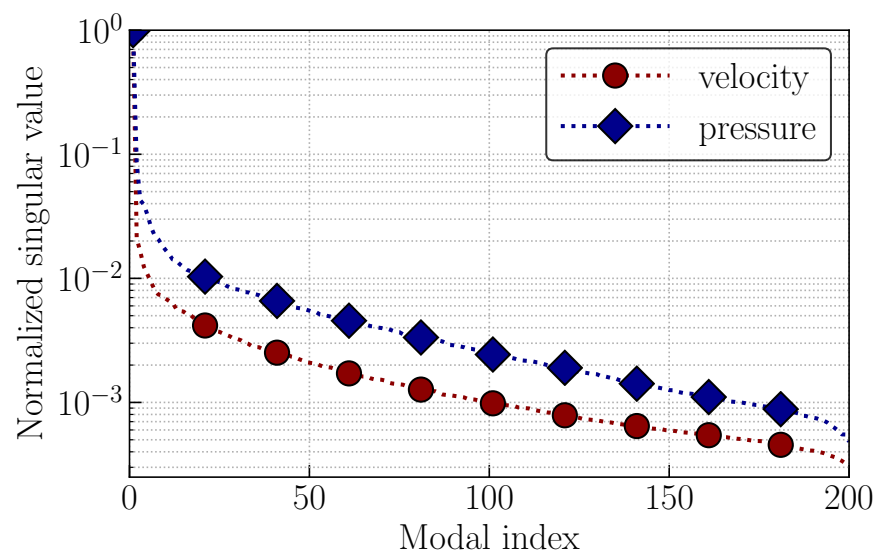

Figure 3: Normalized singular values (SV) for streamwise velocity and pressure snapshots. SV are arranged in descending order. The absence of any gaps in the plots suggests no specific truncation rank for the SV-based reduced models.

For the time integration, a constant time step is set to $\Delta t=10^{-5}$ sec in order to keep the Courant number under the threshold of 0.5 . The flow around the sphere is completely developed after about 80 characteristic times $D / U_{0}$.

\subsection{Modal decomposition}

In this section, we report the numerical results concerning the modal decomposition of the full order snapshots. This phase is particularly useful in order to have an insight onto the dominant structures and on the frequencies hidden in the full order dynamical system. We report both an analysis on the eigenvalue decay which is associated with the Kolmogorov width, and the modal representation which permits to visualize the turbulent structures associated with each mode. Moreover, we analyse the time evolution of the temporal coefficients in order to identify the time frequencies associated with each mode. All the computations have been carried out using the PyDMD Python package 24] and the ITHACA-FV library [91, 90].

\subsubsection{Singular values decay}

Figure 3 depicts the first step of the modal analysis applied to the Navier-Stokes fluid dynamic problem considered. The plot shows the magnitude of the normalized singular values (SV) obtained from the SVD factorization of the snapshot matrix, obtained both for the streamwise velocity field component and for the pressure field. In the diagram - and in all the following discussion - the modes are arranged in descending order according to the corresponding SV magnitude. Typically, a presence of SV magnitude gaps in such plot provides an indication of a convenient truncation rank for the modal analysis. In the present case, a steady and continuous decay is observed after a steep slope corresponding to the first 15 to 20 modes. Thus, the absence of SV magnitude gaps in the higher frequencies region suggests that, for both fluid dynamic fields considered, there is no specific truncation rank for the modal analysis.

\subsubsection{Modal representation}

As shown, the SV magnitude observation is not resulting in an obvious indication of the modal truncation rank. To start understanding the effect of modal truncation rank on the fluid dynamic solution accuracy, we then resort to considerations based on the spatial and time frequencies which need to be reproduced in the hydro-acoustic simulations.

Figure 4 depicts a set of three dimensional modal shapes resulting from the longitudinal velocity field DMD and POD modal decomposition, respectively. The purple diagrams represent isosurfaces passing through the field data points of the DMD modes at value $-15 \times 10^{-5} \mathrm{~m} / \mathrm{s}$, while the olive color plots refer to isosurfaces of POD modes at value $6.5 \mathrm{~m} / \mathrm{s}$. For each modal decomposition methodology, the images in the Figure are arranged in tabular fashion and refer to modes 1, 2, 

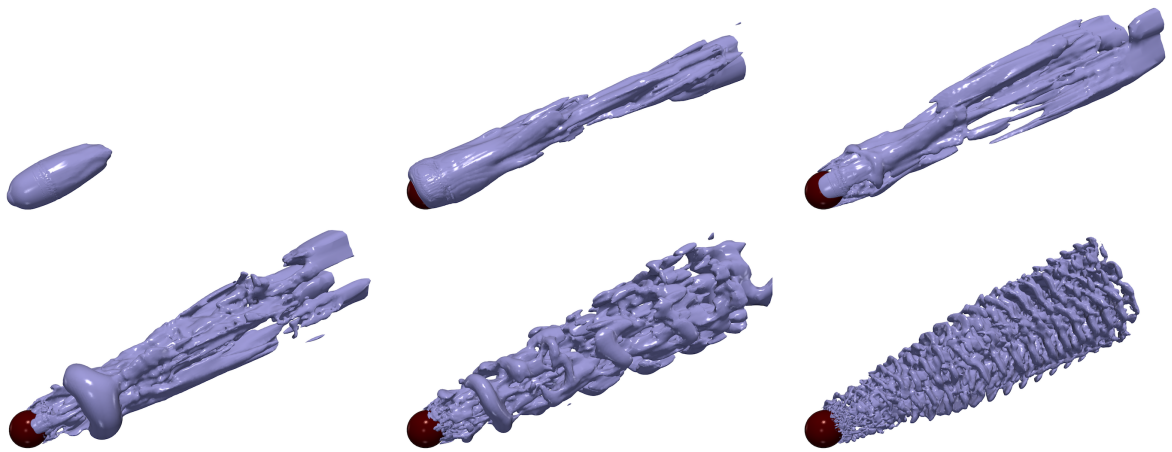

Velocity (POD)

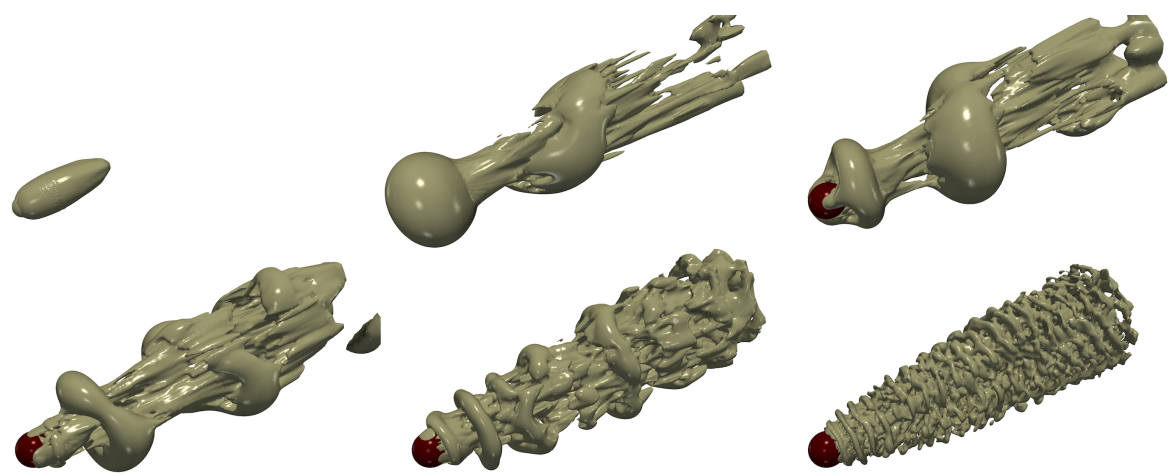

Figure 4: Isosurfaces of the DMD modes (purple color) at value of $-15 \times 10^{-5} \mathrm{~m} / \mathrm{s}$ versus the isosurfaces of POD modes (olive color) at value of $6.5 \mathrm{~m} / \mathrm{s}$ for the streamwise velocity field. Modes: $1,2,4,8,36$, and 128 .

4 on the first row, and $8,36,128$ on the second one. The plots suggest that the DMD modal shapes present a more pronounced tendency to be organized according to spatial frequencies with respect to the POD modes. In fact wider turbulent structures are only found in the very first DMD modes, while in the case of POD modes they can be identified also in higher rank modes, among higher frequency patterns. Along with this tendency, the turbulent structures associated with the low rank DMD modes also appear organized in longitudinal streaks, and gradually become more isotropic for higher rank modes. It should be pointed out though that despite the fact that POD modes are in general not designed to separate the contributes of single harmonic components, the modal shapes obtained for the longitudinal velocity do appear to be at least qualitatively correlated to spatial frequencies. In fact, by a qualitative standpoint, the spatial frequencies appearing in the plots corresponding to higher modes are in general higher with respect to those associated to the first modes.

Similar considerations can been drawn from the observation of similar plots corresponding to the modal decomposition of the pressure fields, presented in Figure 5. Also in this case, the purple plots refer to isosurfaces passing through all data points of value $-5 \times 10^{-4} \mathrm{~m}^{2} / \mathrm{s}^{2}$ of DMD modal shape functions, while the olive diagrams refer to isosurfaces of POD modes at the value $300 \mathrm{~m}^{2} / \mathrm{s}^{2}$. The plots are again arranged, for each decomposition methodology considered, in tabular fashion portraying modes $1,2,4$ on the first row, and 8, 36, 128 on the second one. The pressure modes associated with both methods seem again qualitatively arranged according to spatial frequency content. As previously observed for the longitudinal velocity modes, the DMD modes appear more closely correlated to spatial frequencies, and present a less isotropic appearance with respect to 
Pressure (DMD)
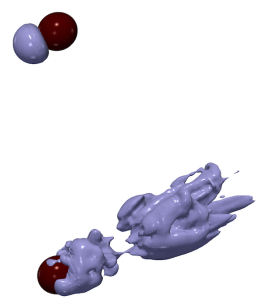

Pressure (POD)
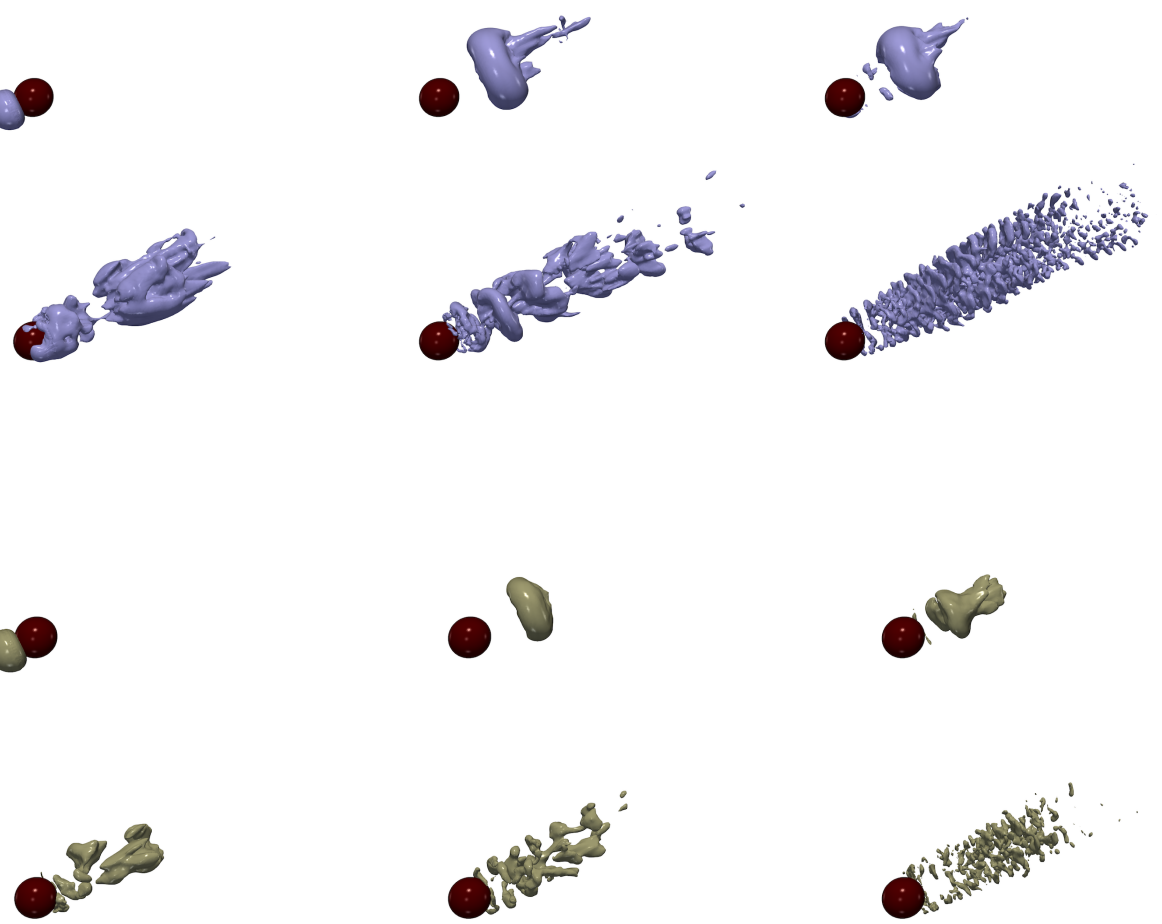

Figure 5: Isosurfaces of the DMD modes (purple color) at value of $-5 \times 10^{-4} \mathrm{~Pa}$ versus the isosurfaces of POD modes (olive color) at value of $300 \mathrm{~Pa}$ for the pressure field. Modes: 1, 2, 4, 8, 36 , and 128 . 

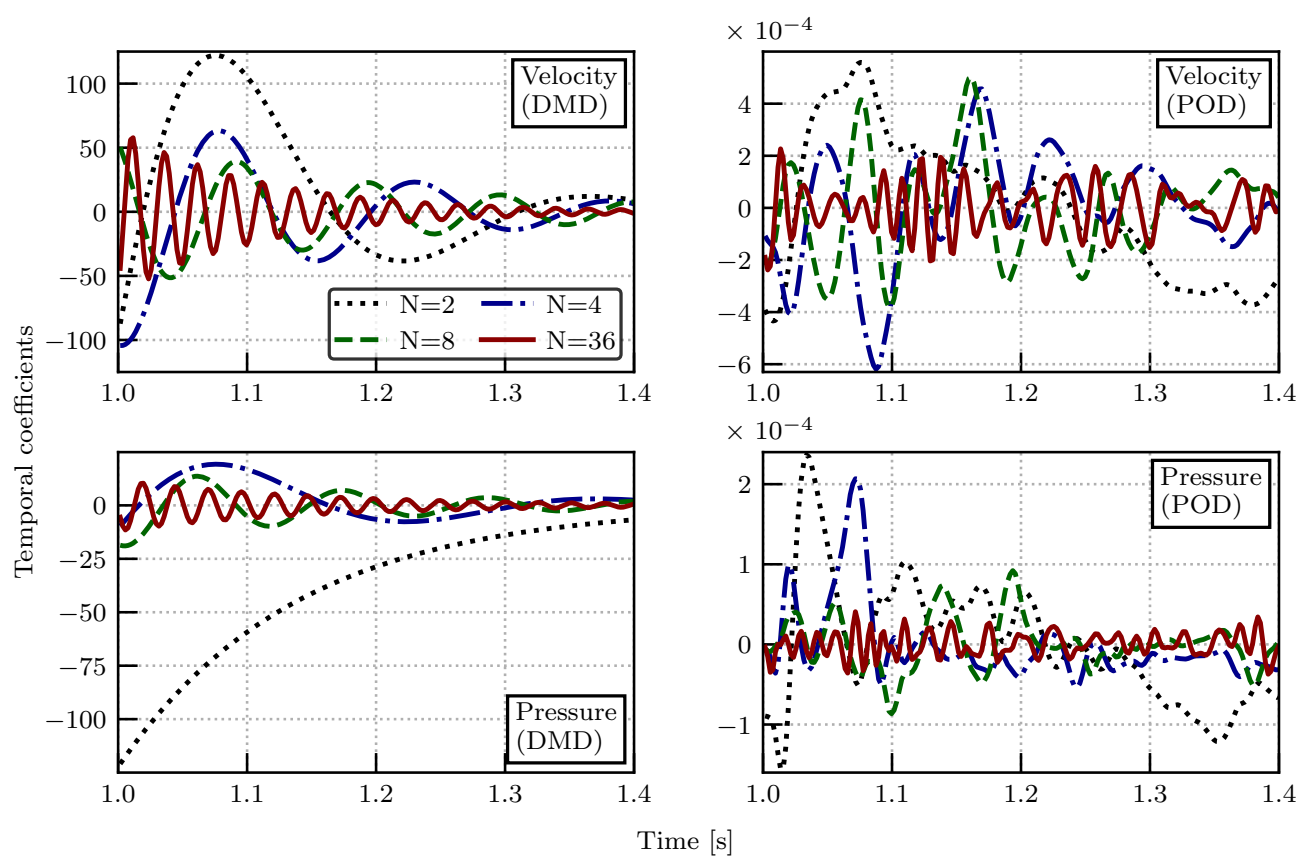

Figure 6: Temporal coefficients associated to the DMD and POD (left to right) modes number 2, 4,8 , and 36 of the streamwise velocity and pressure data (top to bottom).

their POD counterparts.

\subsubsection{Associated coefficients}

After having characterized the spatial frequency content of different POD and DMD modes, we now want to analyse the time frequencies associated to each mode. To do this, we decompose each snapshot into its modal components and observe the time evolution of the modal coefficients. In fact, as the POD and DMD modes generated from the snapshots are constant in time, the corresponding modal coefficients must depend on time to allow for the reconstructed solution to reproduce the correct time variation.

The four plots presented in Figure 15 show, the temporal evolution of the modal coefficients associated, respectively, to the DMD decomposition of the longitudinal velocity field (top left), to the POD decomposition of the longitudinal velocity field (top right), to the DMD decomposition of the pressure field (bottom left) and to the POD decomposition of the pressure field (bottom right). Each diagram reports four lines referring to the coefficients of modes 2, 4, 8, 36 .

By a qualitative perspective, the diagrams in Figure 15 suggest that DMD and POD modal coefficients are strongly correlated with the time frequencies. In fact, higher frequency harmonics appear in the time evolution of higher order modal coefficients, which are not observed in lower ones. As expected, also in this case the frequency-mode association is definitely stronger for DMD modes, in which a single dominant harmonic can be identified in correspondence with each modal coefficient. As for POD, the respective frequency content seems to cover wider set of harmonics, associated with higher frequencies as higher modes are utilized.

\subsection{Flow fields reconstruction}

A first aim of this work is to assess whether the proposed DMD and POD algorithms, are able to accurately reproduce the full order model solutions. A first step in such assessment will be that of checking the effectiveness of the SVD based modal decomposition strategies of the model reduction algorithm considered. In particular, the reconstructed solution convergence to the snapshots considered will be discussed both through the visualization of single snapshots flow fields and by presenting convergence plots of error averaged among snapshots. Finally, we will present similar plots for the solution predicted by means of both DMD and PODI. 

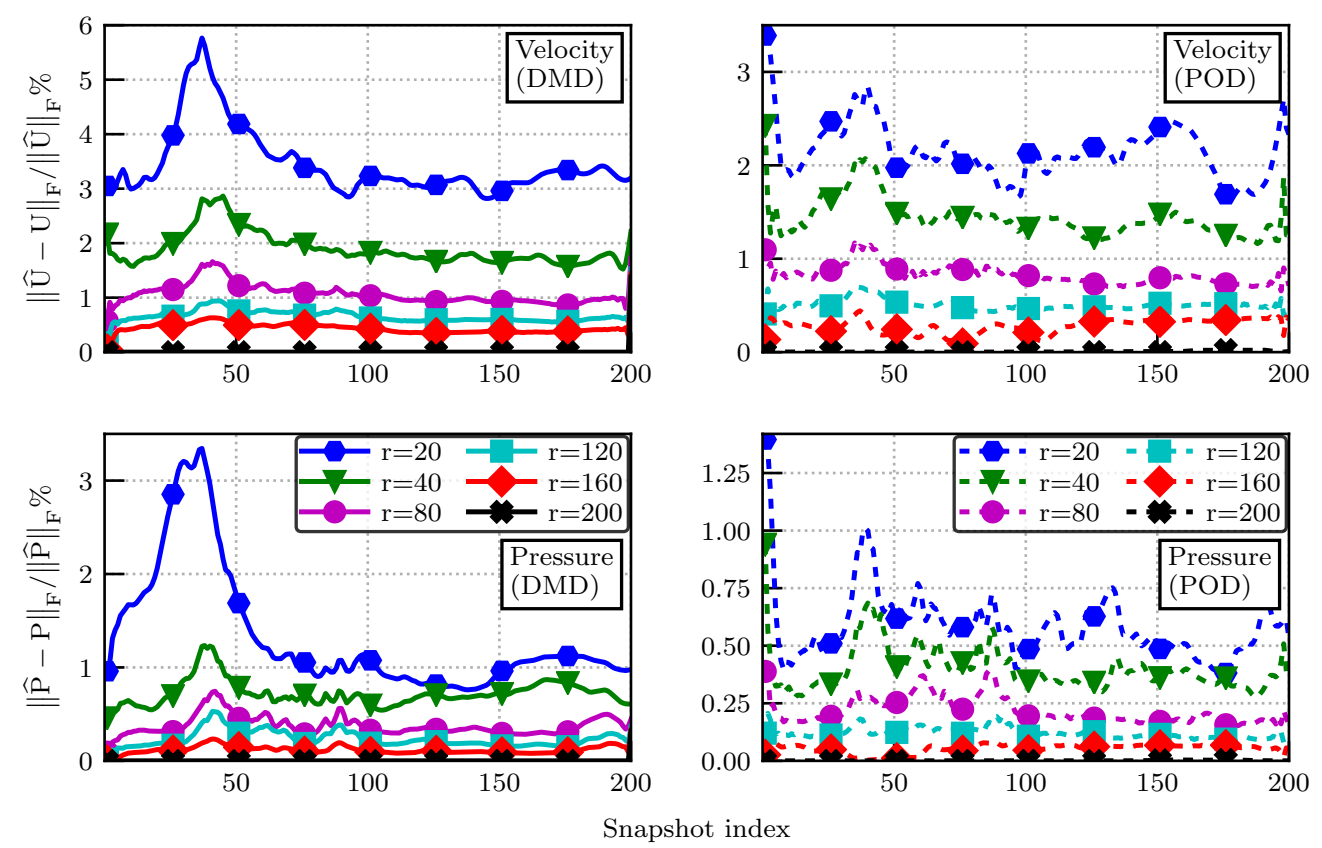

Figure 7: Relative error percentage in the Frobenius norm for the velocity and pressure (top and bottom, resp.) reconstructed fields using the DMD and POD (left and right, resp.) modes. As expected, reconstruction accuracy converges with higher SVD truncation rank (r).

Figure 7 presents a first evaluation of the effectiveness of the DMD and POD modal decomposition algorithm in reducing the number of degrees of freedom of the fluid dynamic problem. The results refer to a reconstruction exercise in which the LES solutions at all the time steps have been used for the modal decomposition. The curves in the plots indicate the relative reconstruction error at each time step for both velocity (top plots) and pressure (bottom plots) when a growing number of modes are considered. Such relative reconstruction error is computed as the Frobenius norm of the difference between the LES solution vector and the reconstructed one, divided by the Frobenius norm of the LES solution. We here remark that to make the velocity and pressure fields error values comparable, the gauge atmospheric pressure value in the simulations has been set to one. A null value would in fact result in lower LES solution norm, leading in turn to large pressure relative errors compared to the velocity ones, even in presence of comparable absolute errors. In Figure 7, the two plots on the left refer to DMD reconstruction results, while the ones on the right present the POD reconstruction error. As can be appreciated, for both modal decomposition methods the errors presented follow the expected behavior, and reduce as a growing number of modes is used in the reconstruction, until machine precision error is obtained when all the 200 modes available are used to reconstruct the 200 snapshots. More interestingly, the data indicate that both for DMD and POD, a number of modes between 80 and 120 leads to velocity and pressure reconstruction errors which fall under $1 \%$ across all the time interval considered. It is worth pointing out that, compared to most typical low Reynolds and RANS flows applications of DMD and POD methodologies, such convergence rate is rather slow, as higher number of modes are needed to obtain comparable accuracy. This should not surprise, as LES resolves more turbulent structures than the aforementioned models, resulting in higher spatial frequencies which in turn require a higher number of modal shapes to be accurately reproduced. The peak we see for smaller rank truncations around the 40-th snapshot for the DMD reconstruction is justified by the fact that those snapshots present a wider range of frequencies thus we need more DMD modes to properly reconstruct them. Finally, the plots suggest that the reconstruction with POD modes leads to errors that are slightly lower to the corresponding DMD errors. In fact, the results consistently show that for both the pressure and the velocity fields, the POD reconstruction error is approximately half of the DMD error obtained with the same amount of modes. 


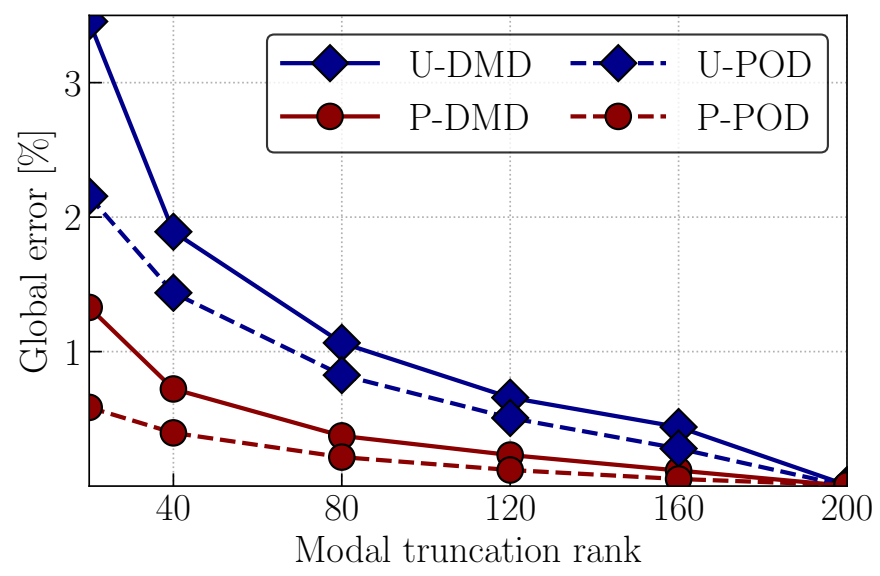

Figure 8: Global error of the reconstructed velocity and pressure fields versus SVD truncation rank. The error denotes spatio-temporal averaging of the flow field data, first by evaluating relative error in the Frobenius norm, then by averaging over all the snapshots.

\subsubsection{Global error}

Figure 8 presents further verification of the modal reconstruction accuracy. The curves in the plot represent the percentage modal reconstruction error — computed in Frobenius norm and averaged among all snapshots - as a function of the number of modes considered in the reconstruction. The black and red curves confirm that the reconstructed velocity and pressure fields, respectively, converge to the corresponding LES fields as the number of modes is gradually increased. Moreover, these results indicate that an efficient reconstruction, characterized for instance by a $1 \%$ relative error, would require more than 80 modes for the velocity fields, and more than 30 for the pressure fields. These values are higher than those typically observed for RANS and low Reynolds simulations, probably due to the higher spatial frequencies typically found in eddy-resolving solution fields.

\subsubsection{Fields visualization}

The error indicators considered in the previous sections are extremely useful in confirming that the reconstructed solution is globally converging to the LES one as the number of modes is increased. Yet, they offer little information on the error distribution in the flow field, and the impact of the reconstruction on local flow characteristics of possible interest. In particular, for the test case considered in the present work, it is quite important to assess whether the reconstruction error does not alter the flow in proximity and in the wake of the sphere, as such regions are crucial both to the evaluation of the fluid dynamic forces on the sphere and to the acoustic analysis. To this end, in the present section we present a series of visualization of the reconstructed flow fields, which are compared to their LES counterparts.

Making use of the Q-criterion, defined as $Q=0.5\left(\|\boldsymbol{\Omega}\|^{2}-\|\mathbf{S}\|^{2}\right)$ with $\boldsymbol{\Omega}$ and $\mathbf{S}$ denoting the vorticity and strain rate tensors respectively, Figure 9 depicts the turbulent structures characterizing the flow in the wake region past the sphere. By definition, a positive value of $Q$ implies relative dominance of the vorticity magnitude over the strain rate [38. Here, a positive value of $10^{4} 1 / \mathrm{s}^{2}$ is chosen to generate isosurfaces which pass through all data points holding this value. In the figure, the top plot refers to the original LES solution obtained at the last snapshot of the dataset, while the centered and bottom plots refer to the corresponding DMD and POD reconstructions, respectively, utilizing 160 modes. The images show that both POD and DMD reconstruction algorithms lead to fairly accurate representation of the turbulent structures shape past the sphere. In fact, the configuration of the wider vortical structures detaching from the sphere appears to be correctly reproduced in the reconstructed solution. As for finer details associated with the smaller turbulent scales, the DMD reconstruction is observed to be in closer agreement with the original LES solution than the POD reconstructed field. Such observation is consistent with findings from [103, 4] in which they demonstrated the superiority of DMD to accurately determine spectral and convective 


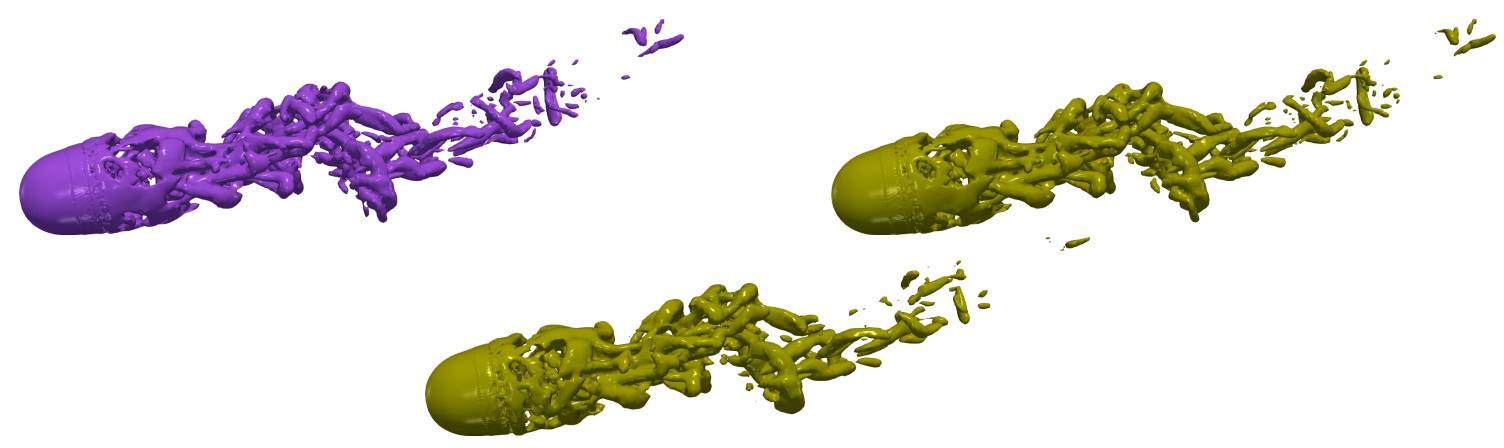

Figure 9: Coherent structures represented by the iso-contours of the Q-criterion at non-dimensional value $Q D^{2} / U_{0}^{2}=4$ for the last snapshot (top), compared with corresponding ones obtained from modal reconstruction with DMD (centered) and POD (bottom) using 160 modes.

information of the vortical structures in wake regions.

For a more significant and quantitative assessment, Figure 10 includes a series of of contour plots representing the instantaneous flow field at time instance corresponding to a maximized relative error, cf. Figure 7. The three plots in the first row represent contours of the LES streamwise velocity component field, and of its DMD and POD reconstructed counterparts, respectively, utilizing 160 modes. At a first glance, the reconstructed fields seem to reproduce the main features of the LES flow. In particular, both the stagnation region ahead of the sphere and the flow detachment past it appear to be correctly reproduced by both modal reconstruction strategies. In addition, the detached vortex, located downstream with respect to the sphere in this particular time instant, is also correctly reproduced. For a better assessment, the two images of the second row represent contours of the local error for the DMD and POD streamwise velocity reconstruction, respectively. Both for the DMD and POD reconstruction, higher local error values are found in the wake region downstream with respect to the sphere. In particular, it observed that the local error peaks in the DMD reconstruction is larger than that for POD. Additionally, the high frequency error pattern and the elevated local error values located in the wake region seem to indicate that, as expected, the modes disregarded in the reconstruction are associated with high spatial frequencies. A similar comparison is presented for the pressure field in the following rows of the figure. The three plots in the third row represent the instantaneous pressure field obtained with LES, and its reconstructions computed with DMD and POD, respectively, for the same snapshot. Again, the full order field appears well reproduced by both DMD and POD reconstruction algorithms, as features like the peak pressure in the stagnation region and the pressure minimum within the vortex detaching past the sphere are correctly reproduced. The plots of pressure reconstruction error for DMD and POD, respectively, presented in the last row confirm that both methods are capable to adequately represent the LES solution. Also here, the disregarded high spatial frequency modes are likely responsible for the high frequency error pattern observed.

\subsubsection{Error statistics in the wake region}

As Figure 10 shows, the highest reconstruction errors in the velocity and pressure fields reconstruction are mostly located in the wake region. Therefore, a follow up analysis is required to quantify the spatial error distribution within such region and to assess the local convergence behavior of both the DMD and POD modal decomposition methodologies. Figure 11 presents a normalized density function plot based on the reconstruction error of both the velocity and pressure fields corresponding to the snapshot illustrated in Figure 10. To generate the plot, the following steps are performed. First, the computational cells in the wake region are identified according to the condition $\left\|\boldsymbol{\omega}_{x}\right\|=\left\|\nabla_{x} \times \boldsymbol{U}\right\|>1.0$ with $\boldsymbol{\omega}_{x}$ denoting the streamwise vorticity component. Second, the arrays corresponding to the velocity and pressure error fields in the wake region are computed, i.e. $e_{w}^{U}=\left(\hat{U}_{w}-U_{w}\right)$ and $e_{w}^{P}=\left(\hat{P}_{w}-P_{w}\right)$ wherein the subscript denotes the wake cells. Third, to account for variations in the grid resolution, the mentioned error fields within the wake region are weighted by the corresponding normalized cell volumes $V_{c}^{\text {norm }}$, i.e. $\bar{e}_{w}=\left(\sum_{i=1}^{n} V_{c, i}^{\text {norm }} e_{w, i} / \sum_{i=1}^{n} V_{c, i}^{\text {norm }}\right)$ wherein $n$ is the cell count in the wake. Finally, the error interval for both the velocity and 


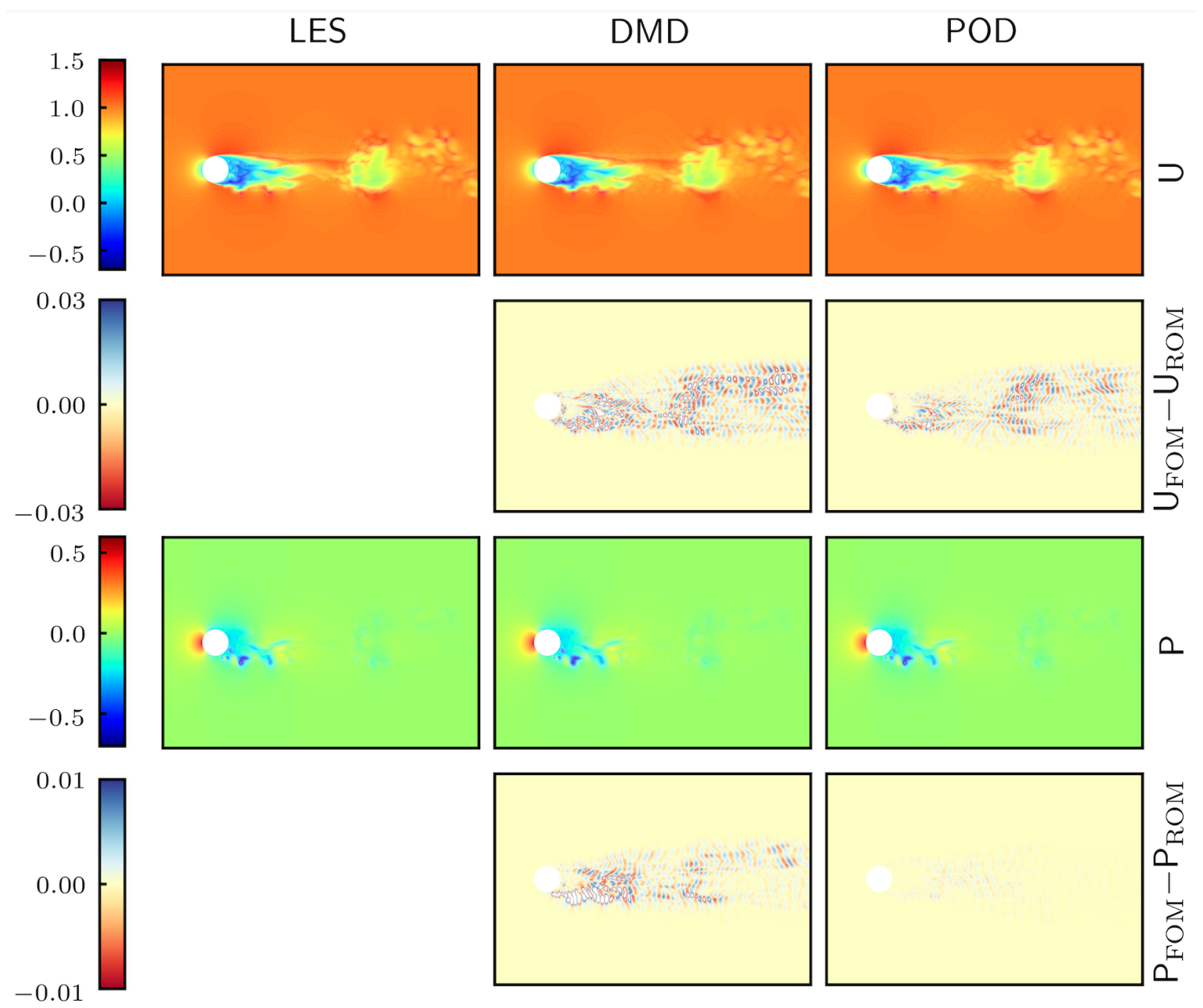

Figure 10: Left to right: LES, DMD and POD reconstructions with $N=160$ modes. Top to bottom: streamwise velocity, corresponding error fields, pressure, corresponding error fields. Instantaneous snapshot is selected based on errors peak. 


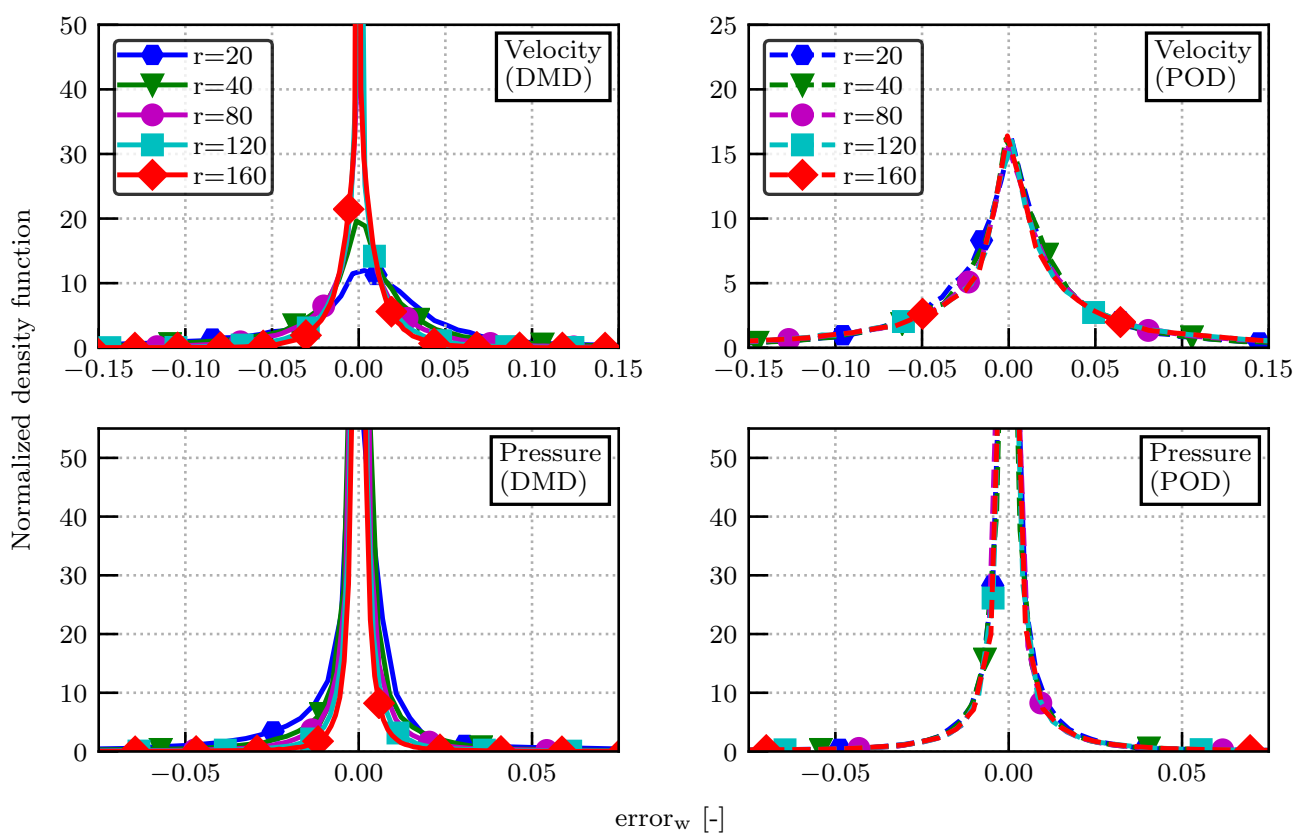

Figure 11: Error statistics of velocity and pressure field data of selected snapshot corresponding to maximized relative error. Sampled data points are conditioned by $\left\|\boldsymbol{\omega}_{x}\right\|=\left\|\nabla_{x} \times \boldsymbol{U}\right\|>1.0$ to identify the wake region. Resulting density function is weighted by normalized cell volumes. Presented plots correspond to various modal truncation ranks (r).

pressure data are uniformly divided into equal-width bins and the density function (normalized histogram) is plotted for each bin.

The scattered plots highlight differences in the behavior of the two modal reconstruction strategies considered. The diagrams on the left, which refer to the DMD results for velocity (top) and pressure (bottom), show in fact a clear error reduction as the modal truncation order is increased. The curves corresponding to growing truncation orders tend to get closer to the vertical axis as the error, displayed on the horizontal axis, is progressively reduced. The same convergence rate cannot be observed in the POD plots on the right, as both the velocity (top) and pressure (bottom) reconstruction error statistical distribution curves appear less affected by an increase of the modal truncation order. Again, this observation can be explained in the light of the DMD theory and the ability of its modes to be organized according to the field spatial frequencies, unlike POD ones which can instead become contaminated by uncorrelated structures, according to the claim reported in 103 .

It is worth pointing out that for image definition purposes, the left and right tails of Figure 11 have not been reported. Yet, a cross comparison with Figure 10 readily suggests that POD results showed lower error margin - hence narrower tails - in this regard. Therefore, we could infer that higher DMD modes are capable to capture more frequencies in the wake, resulting in lower mean error but higher peak errors compared to POD modes.

\subsection{Fields mid cast using DMD and PODI}

The previous sections are focused on assessing the accuracy of the POD and DMD modal decomposition strategies. The reconstruction results confirmed that both methods can be considered effective tools for the reduction of the degrees of freedom of the fluid dynamic problem. We now want to analyse the ability of the data-driven DMD and PODI reduced order models considered in this work, in predicting the LES solution at time steps that are not included in the original snapshot set. In particular, throughout the remaining analysis of this work, the original 200 LES snapshots are decomposed into two sets, one set comprises the 100 odd snapshots from which they are used to train the ROMs and are called the train dataset hereafter, while the remaining even snapshots within the temporal interval of the train dataset are contained in a test dataset, and are used to compare the full order solution with the DMD and PODI model prediction results (i.e. 
Table 1: ROM performance at various modal truncation rank. Data compression level is defined as $\left(\mathrm{GB}_{\mathrm{FOM}} / \mathrm{GB}_{\mathrm{ROM}}\right)$ with $\mathrm{GB}_{\mathrm{FOM}}=74$. Speedup is defined as $\left(\mathrm{CPU}_{\mathrm{FOM}} / \mathrm{CPU}_{\mathrm{ROM}}\right)$ with $\mathrm{CPU}_{\mathrm{FOM}}=$ $2.4 \times 10^{5}$ sec. Compression level is averaged between DMD and PODI.

\begin{tabular}{cccccc}
\hline \hline Rank & \multicolumn{2}{c}{ POD cumulative energy } & \multicolumn{2}{c}{ Speedup } & Compress. \\
$(\mathrm{r})$ & $\mathrm{U}$ & $\mathrm{P}$ & $\mathrm{DMD}$ & PODI & level \\
\hline \hline 10 & 0.999992 & 0.994093 & 29.29 & 35.67 & 19.995 \\
20 & 0.999996 & 0.997076 & 28.46 & 32.46 & 9.9973 \\
40 & 0.999998 & 0.998735 & 26.41 & 27.93 & 4.9986 \\
60 & 0.999999 & 0.999295 & 25.41 & 24.68 & 3.3324 \\
80 & 0.999999 & 0.999571 & 23.58 & 22.48 & 2.4993 \\
100 & 1.000000 & 1.000000 & 23.18 & 20.46 & 1.9995 \\
\hline \hline
\end{tabular}

ROM prediction dataset).

Before progressing with analysis, a summary on the POD energetic content, as well as the data compression level and computational speedup for the considered DMD and PODI data-driven models at various modal truncation ranks is listed in Table 1. Here, the kinetic energy content within a ROM is described by cumulative sum of the POD eigenvalues. The compression level, taken as the arithmetic mean between DMD and PODI in the table, considers the size ratio of the FOM data (train and test datasets, totalling $74 \mathrm{~GB}$ ) to the ROM data (spatial modes of the train dataset, and continuous representation of the temporal dynamics computed via time integration or cubic spline interpolation for DMD or PODI, respectively). Speedup is defined as $\left(\mathrm{CPU}_{\mathrm{FOM}} / \mathrm{CPU}_{\mathrm{ROM}}\right)$ with $\mathrm{CPU}_{\mathrm{FOM}}=2.4 \times 10^{5} \mathrm{sec}$ corresponding to the time required to solve for the 200 snapshots and to write out the train dataset, while $\mathrm{CPU}_{\mathrm{ROM}}$ is the time needed to 1) perform modal decomposition on the train dataset, 2) extract the associated dynamics with a continuous representation, and 3) write out the prediction dataset in OpenFOAM format.

It is worth noting that, since time is the considered parameter in the present ROM procedure, an offline phase still requires solving for the same temporal window as in the FOM solution, in order to obtain the train dataset. Nevertheless, a computational gain in the generated ROM can be still attained, since the less amount of stored data (i.e. few modes and associated dynamics) compared with FOM are utilized for a swift construction of the fluid dynamic fields at arbitrary sample points. Such procedure is considered a lot faster and more economic than recomputing the simulation to write out the fluid dynamic field data at those sample points. Indeed, an extension of the present procedure to consider additional parameters while performing, for instance, multiparameter interpolation using PODI, would result in a further speedup since the offline phase would solve a full order solution only for a subset of the parameter combinations in the parameter space, hence saving up complete FOM computations from being performed. Such multiparameter investigation is considered a follow up study of this work. To this end, now we progress the analysis by considering the temporal evolution of the error due to ROMs prediction.

\subsubsection{Prediction error analysis}

The first test presented is designed to assess the predictive accuracy of the DMD and PODI methodologies described in this work. Figure 12 depicts the results obtained with the ROM methodologies applied in such alternate snapshots arrangements as previously described. The top plots report the percentage Frobenius norm error between the DMD and PODI predictions with respect to the LES solution for the longitudinal component of the velocity, while the bottom diagrams present similar error norms corresponding to the pressure field. The left plots refer to the DMD results, while the right ones depict the PODI errors. We point out that, the - significantly lower - error values corresponding to the train dataset have been omitted for clarity. Finally, the different colors in the plots indicate growing number of modes considered in the DMD and PODI prediction, up to a maximum of 100 .

The results show a substantial convergence of the data-driven reduced model solutions to the full order one. In particular, selecting a number of modes between 80 and 90 results in errors lower than $2 \%$ on the velocity field for most time steps in both DMD and PODI methods. As for the pressure field, the bottom left diagram in Figure 12 shows that $1 \%$ error goal can be obtained with an even slightly lower amount of DMD modes. It must be pointed out though, that the DMD solution 

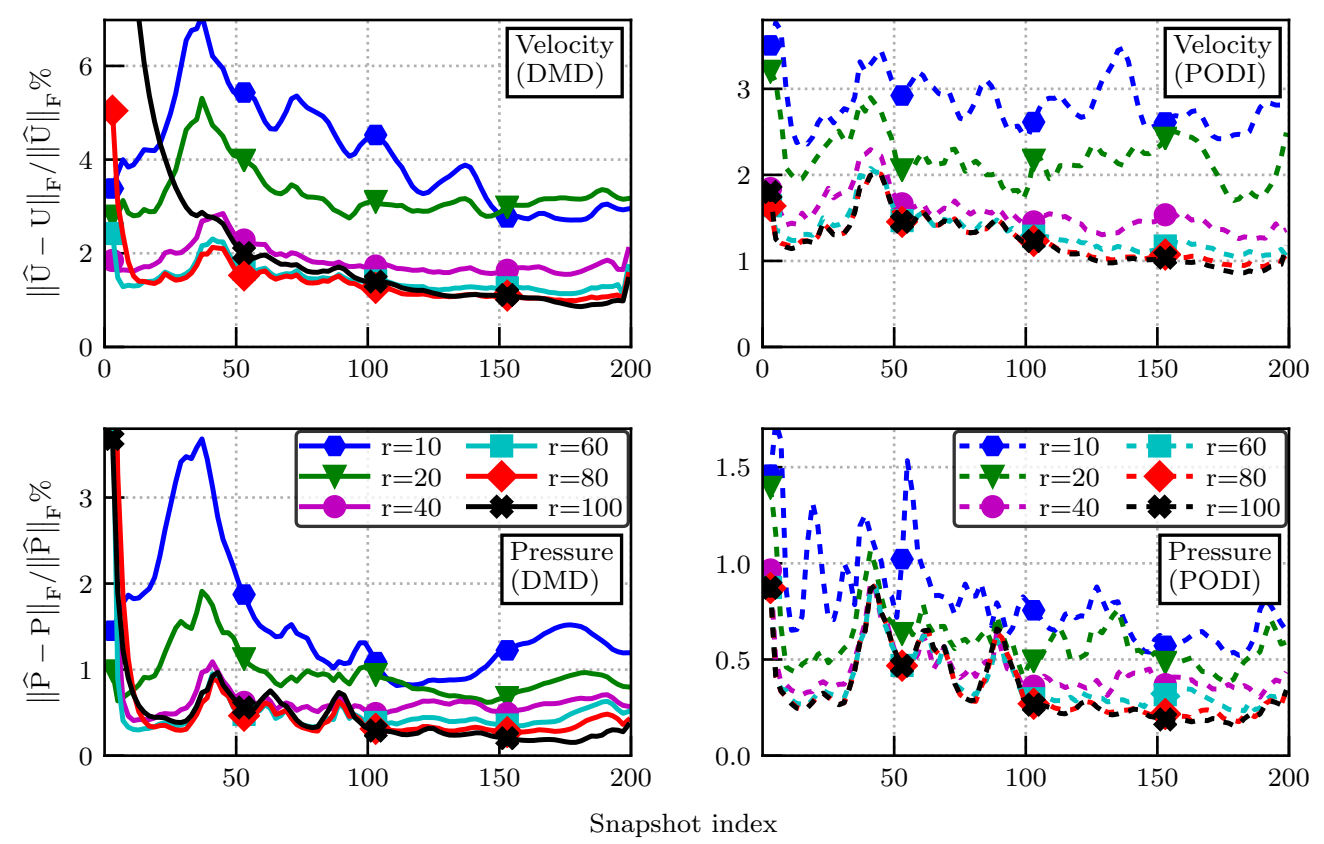

Figure 12: Relative error percentage in the Frobenius norm for velocity and pressure (top and bottom, resp.) fields, where half sample rate snapshots are used to train the reduced model, DMD (left) or PODI (right), for particular truncation (r) while predicting the intermediate snapshots. Plots show only the snapshot-wise prediction error, while disregarding errors in the training set, which is almost null.

in the very first time steps is not converging to the LES one, and the error grows as the number of modes is increased. This behavior, which could be related to the high frequencies introduced by the higher DMD modes added to the solution, is currently under further investigation. Aside from the first few time steps, it is generally observed that PODI errors are consistently lower by a factor two with respect to the DMD ones, especially in the cases utilizing fewer modes. It is worth pointing out that a direct comparison between the PODI plots in Figure 8 and those in Figure 12 can indirectly result in a possible estimate of the interpolation error associated with the PODI strategy. The plots suggest that the effect of interpolation on the global spatial error at each time step is rather low, as the errors in Figure 12 present the same behavior and are not significantly higher than the ones obtained with pure reconstruction.

Now, to summarize the performance of each ROM with respect to the modal truncation level, a global spatio-temporal error is measured against growing number of modes, as depicted in Figure 13. In particular, similar to the previous analysis in Figure 8, the mean absolute error is evaluated for the percentage Frobenius norm spatial error for all the prediction dataset, cf. Figure 12. Here, it is observed that, for both the velocity and pressure predictions, a significant reduction in the global error is achieved in the PODI models with respect to DMD up to a utilization of 60 modes, after which the global error becomes comparable between both ROMs. Additionally, a finite global error is noted in the figure even when a full modal rank is utilized. Indeed, such observation should not be surprising since half the dataset is only employed to train both ROMs while interrogating the remaining sample points. This is not the case in pure field reconstructions, cf. Figure 8, where the global error vanishes with a full modal rank since dynamic and spectral information become entirely recovered.

\subsubsection{Coherent structures}

The prediction error indicators considered in the previous section indicate whether the reduced models solution is globally converging to the LES one as the number of modes is increased. We now resort to flow visualizations to obtain better information on the error distribution in the flow field and on the reduced models performance in reproducing local flow characteristics of possible 


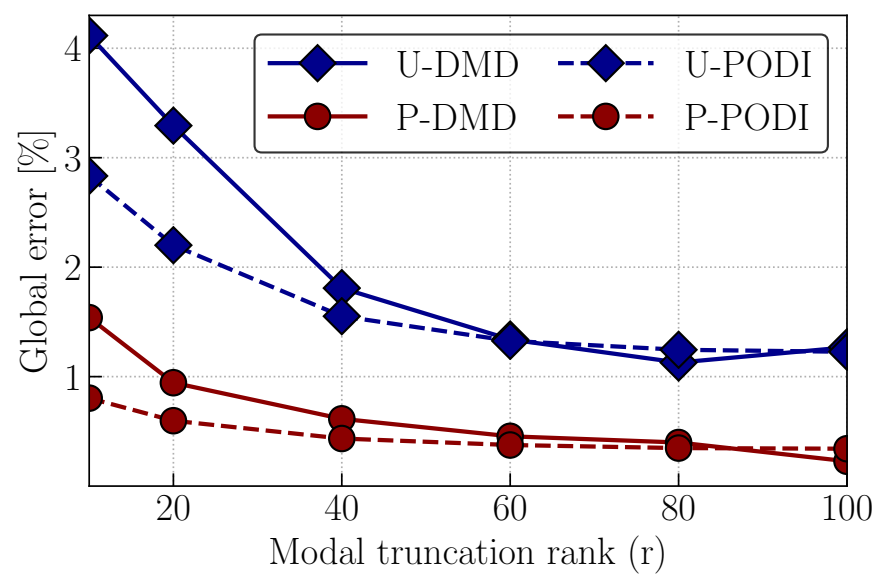

Figure 13: Global error of the prediction dataset versus SVD truncation rank. The error denotes spatio-temporal averaging of the flow field data, first by evaluating relative error in the Frobenius norm, then by averaging over all the snapshots.

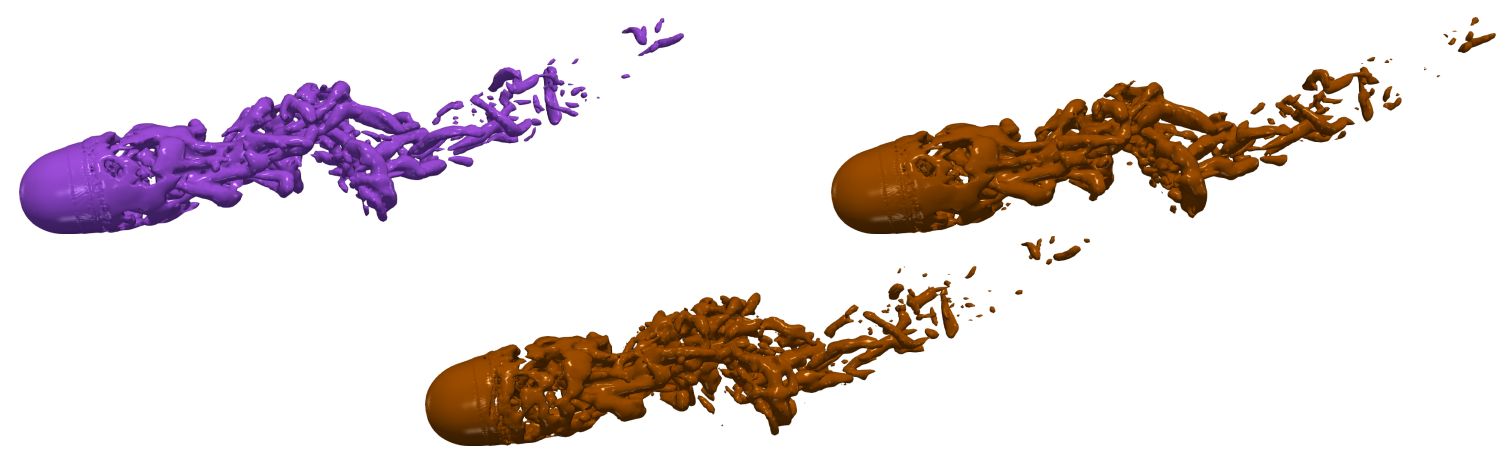

Figure 14: Coherent structures of the last snapshot in the prediction dataset, represented by the isocontours of the $\mathrm{Q}$-criterion at non-dimensional value of $Q D^{2} / U_{0}^{2}=4$, compared with corresponding ones obtained from DMD-100 (centered), and PODI-100 (bottom).

interest.

Again, making use of the Q-criterion isosurfaces at value $Q D^{2} / U_{0}^{2}=4$, Figure 14 portrays the turbulent structures characterizing the wake flow past the sphere. The top plot refers to the original LES solution obtained at the last snapshot of the prediction dataset, while the centered and bottom plots refer to the respective DMD and PODI predicted solution utilizing 100 modes. Also in this case, the images show that both DMD and PODI reduced order models allow for rather accurate reproduction of the turbulent structures past the sphere. For both ROM solutions, the main vortical structures detaching from the sphere appear in fact very similar to those of the original LES flow field. Finer details associated with smaller turbulent scales are also in good agreement, hence suggesting that errors in the PODI time interpolation and DMD time integration are not significantly higher with respect to the reconstruction error analysed earlier.

\subsubsection{Drag and lift coefficients}

Besides coherent structures, it is important to assess the performance of data-driven ROMs in capturing hydrodynamic phenomena that could find particular interest in the engineering community. One of these phenomena is the drag and lift forces on the sphere surface. First, pressure-induced drag and lift forces are evaluated by integrating the pressure in the streamwise and the flow-normal directions, respectively, over the sphere surface. Then, the pressure forces are normalized by the dynamic pressure acting on the sphere, with a sphere projected cross-sectional area being considered for the reference area, to compute coefficients of drag $\left(C_{D}\right)$ and lift $\left(C_{L}\right)$. The temporal evolution of both coefficients on the sphere surface is depicted in Figure 15 for various ROM predictions compared with the FOM. The presented plots show that both $C_{D}$ and $C_{L}$ are accurately 

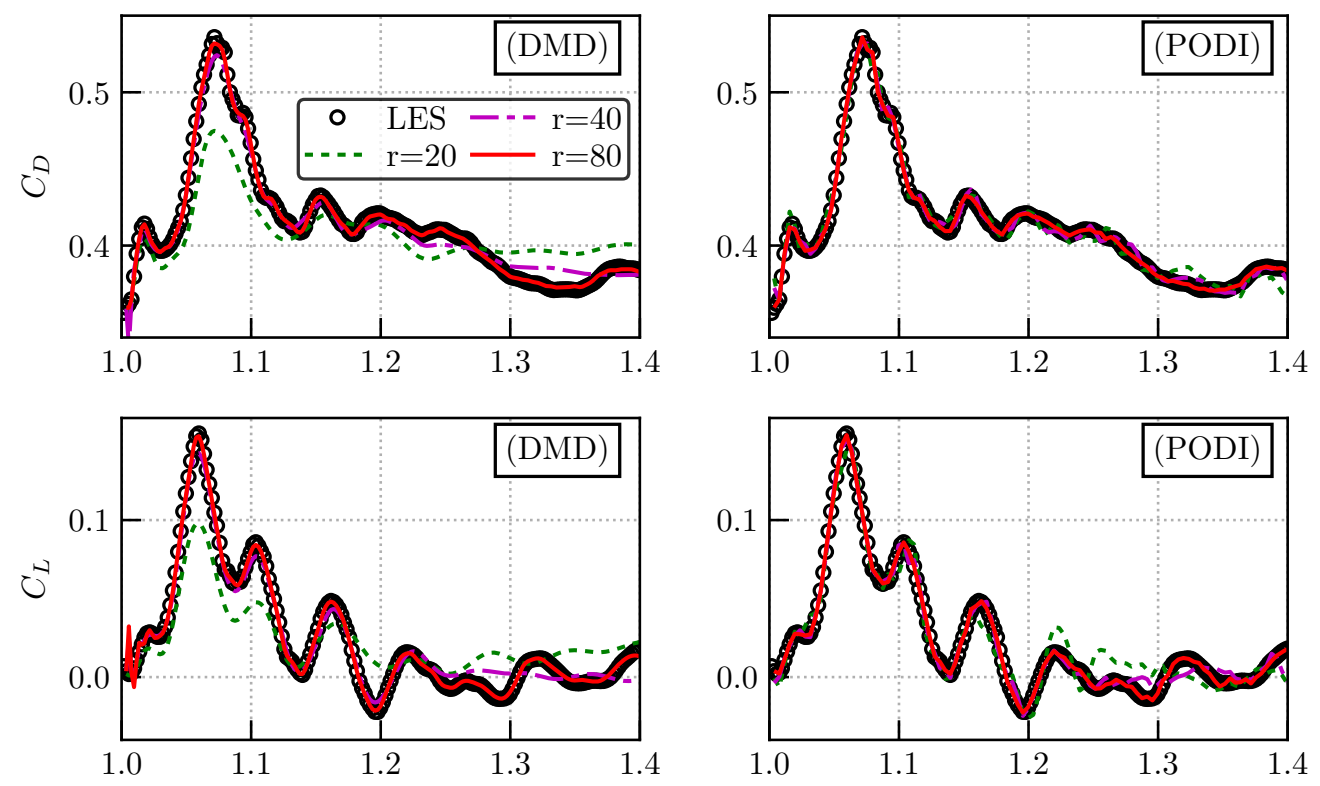

Time $[s]$

Figure 15: Temporal evolution of the drag and lift coefficients corresponding to pressure forces on the immersed sphere surface. The force coefficients are computed for various DMD and PODI models in comparison to FOM data. Presented plots show the predictive performance of both datadriven ROMs on estimating the sphere pressure forces, with noted superiority of PODI predictions over DMD.

predicted while only half the dataset is employed. It is noted that the accuracy of $C_{D}$ predictions is higher in comparison to $C_{L}$ predictions, and that employing as low as 40 modes are able to predict the forces coefficients with a sufficient accuracy. More importantly, the predictive character of PODI models are observed to be superior over DMD models for a particular modal truncation level. The observed discrepancies in the early temporal window of $C_{L}$ predictions via DMD at $r=80$ are related to the poor DMD predictions of the flow fields at early snapshots, as previously noted in the relative error plots, cf. Figure 12. On a general note, the presented data-driven ROMs are able to predict the pressure dynamics on the surface of the immersed sphere while employing considerably lower levels for modal truncation. We would like to highlight that the framework that we are proposing is solely based on input and output quantities. Therefore, instead of computing engineering quantities from the reconstructed flow fields, one could also construct a reduced order model to directly approximate them. In such a case we would expect that the so generated ROM would produce even more accurate results.

\subsection{Spectral analysis}

\subsubsection{Data probes inside and outside the wake region}

A first necessary step in order to evaluate how the frequency content of the FOM solution is reproduced at the ROM level, consists in observing the time dependency of the solution at fixed points in the computational domain. To this end, Figure 16 presents the reduced models results for the time evolution of the streamwise velocity component in correspondence with two different locations of the flow field. In the $2 \times 2$ tabular arrangement of the figure, the top plots correspond to a point located outside of the sphere turbulent wake, while the bottom plots refer to a point inside the turbulent wake. In addition, the two diagrams on the left refer to the DMD results, and the ones on the right report the PODI result. In the plots, the red filled circles refer to the LES result at prediction sample points, while the dash-dotted lines represent the ROM results, which have been drawn for a growing number of modes. Finally, the black continuous line represents the pure DMD and PODI reconstruction result utilizing 160 modes. 

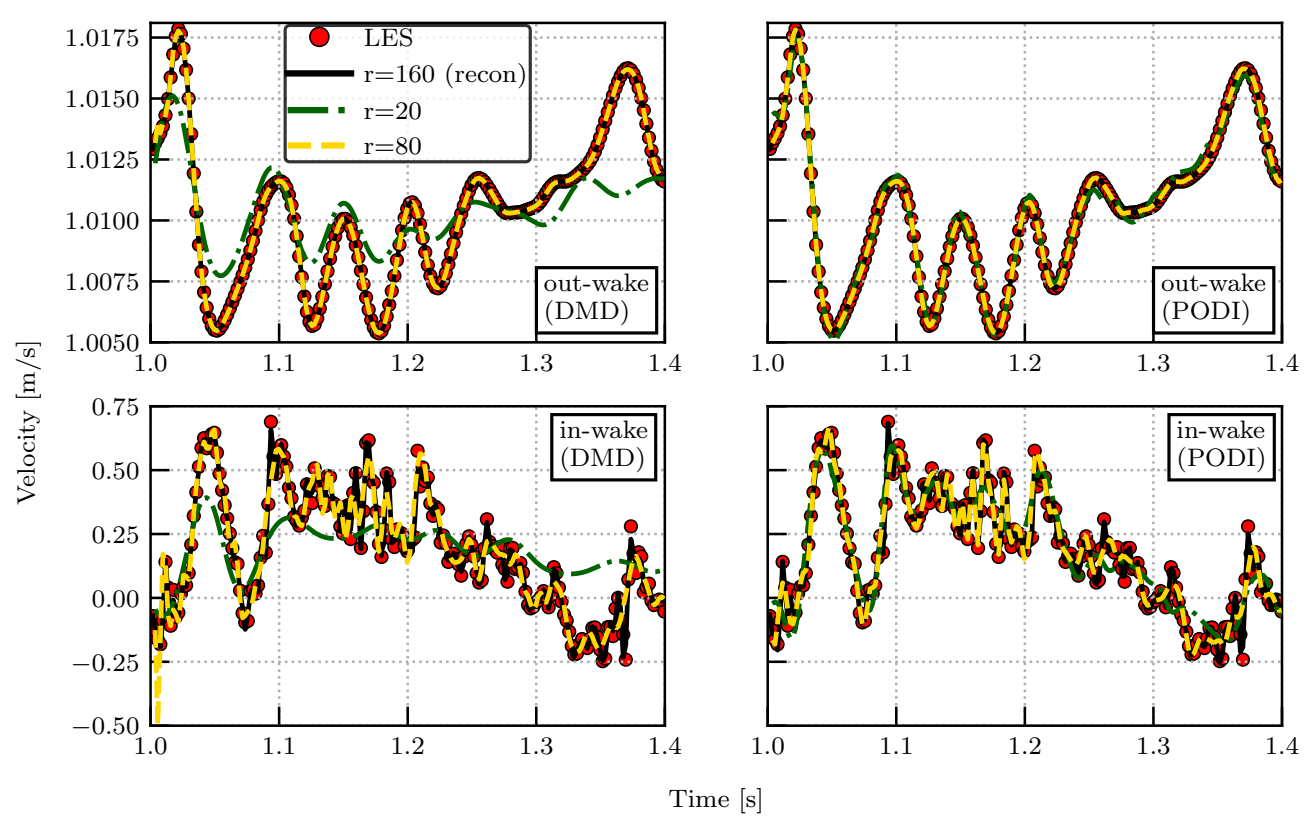

Figure 16: Time history of streamwise velocity signals at fixed points located outside the wake at $\mathbf{x}_{A}=\{2 D, 0,2 D\}$ (top) and inside the wake at $\mathbf{x}_{B}=\{2 D, 0,0\}$ (bottom) for DMD (left) and PODI (right) models. Red symbols represent LES results, while dashed lines represent the ROM results for different SVD truncation ranks (r). Black continuous line represents the reconstructed field utilizing 160 modes.

As expected, the plots show that the velocity variation over time becomes more chaotic in the wake region, due to the high frequency fluctuations associated with the turbulent structures typically observed in such part of the flow domain. All the plots show that, as the number of DMD and PODI modes used is increased, the reduced solution approaches the full order model one. At a first glance, the PODI convergence at the point located outside of the wake appears faster than that obtained with DMD. The reduced PODI solution obtained with 20 modes is, in fact, already rather close to the original time signal, while the corresponding DMD solution is still far from the LES one. The behavior of both DMD and PODI reduced models is clearly more accurate when a higher number of modes is selected. The 80 modes curve obtained with both ROMs is practically indistinguishable from the LES one. Given the small estimate of the time interpolation error, as discussed in subsubsection 3.4.1 the faster PODI convergence observed should depend on the fact that single PODI modes are richer in spatial frequency content than the DMD ones. The low order PODI modes might then already include higher frequencies not contained in the corresponding DMD modes. Such spatial frequency content should finally reverberate in the time evolution of the solution including some higher frequencies also when the only lower order PODI modes are used. Despite these favorable characteristics, when the full order model solution presents even higher frequencies, also the PODI solution requires a higher number of modes to obtain satisfactory accuracy. This is clear by the plots of the time history of the velocity at the point within the wake region. Here, both PODI and DMD reduced result curves become sufficiently close to the original solution one only when 80 modes are considered. This should not come as a surprise, as the in-wake signal presents higher frequency fluctuations due to the wake turbulent structures, which can be fully represented only by including high order modes.

The reduced pressure local time evolution prediction results presented in Figure 17 exhibit the same behavior observed for the reduced velocity field. Also this Figure, in which the plots and the curve colors are arranged as described in Figure 16, suggests in fact that both inside and outside the wake region, employing 80 modes allows for both ROMs to obtain pressure predictions that are sufficiently close to the corresponding LES time signals. Again, the plots also suggest that PODI in the region outside the wake is able to obtain viable pressure predictions with fewer modes. Thus, these results indicate that in presence of fully attached flows, in which wake effects are less dominant, the choice of POD as modal decomposition methodology could result in more economic 

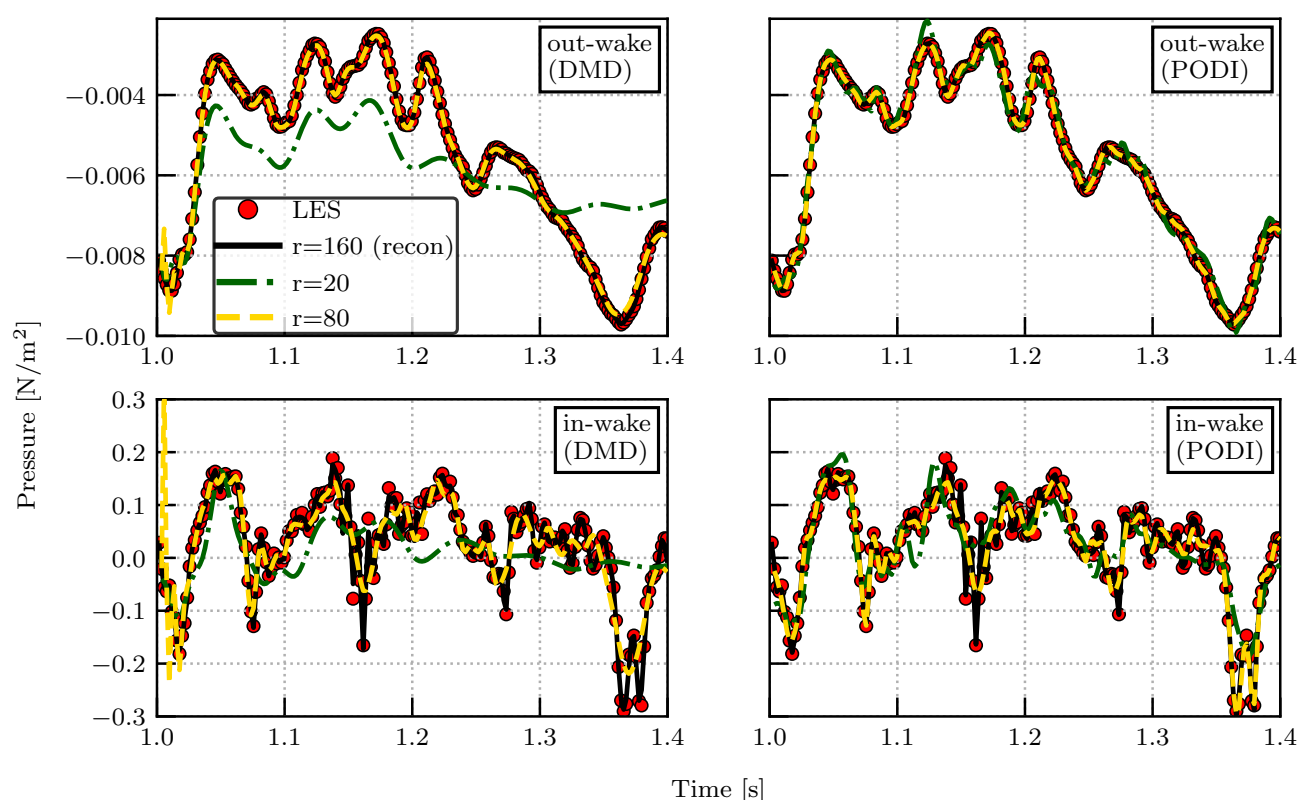

Figure 17: Time history of pressure signals at fixed points located outside the wake at $\mathbf{x}_{A}=$ $\{2 D, 0,2 D\}$ (top) and inside the wake at $\mathbf{x}_{B}=\{2 D, 0,0\}$ (bottom) for DMD (left) and PODI (right) models. Red symbols represent LES results, while dashed lines represent the ROM results for different SVD truncation ranks (r). Black continuous line represents the reconstructed field utilizing 160 modes.

reduced models.

\subsubsection{Fast Fourier transform}

The plots in Figure 16 and Figure 17 seem to indicate that, on a qualitative level, the PODI and DMD solutions are able to recover a growing portion of the full order model frequency content as the number of modes is gradually increased. This aspect should be, of course, investigated in a more accurate way, as the presence of high frequency components in the ROM solution results in their ability to be effective surrogates in acoustic analysis. Thus, we make use of the FFT spectra with the aim of obtaining a quantitative assessment of the impact of the POD and DMD modes considered on the frequency content of the solution. Figure 18 presents the magnitude of the FFT of the local streamwise velocity signals previously presented. Also in this case, the top plot refers to the point located outside of the wake, while the bottom plot refers to the inwake point spectrum. The black, continuous lines refer to the FFT of the local velocity signal obtained with the LES full order model. The red dash-dotted lines represent the spectrum of the signal composed only by the train dataset which, of course, is characterized by half the sampling frequency with respect to the full LES signal. Finally, the green and yellow dashed lines respectively denote the corresponding plots obtained by means of truncated DMD (left panel) and PODI (right panel) models employing 20 and 80 modes each. Figure 19 — which also employs the line color arrangement just described - displays analogous spectral results obtained when the pressure field is considered. We point out that, based on experimental results presented in [76, a $\operatorname{Re}=5000$ flow past a sphere results in the Strouhal number $S_{t}=0.2$ associated with vortex shedding. This corresponds to $f=S_{t} U / D=20 \mathrm{~Hz}$ being $U=1.0 \mathrm{~m} / \mathrm{s}$ and $D=0.01 \mathrm{~m}$. In Figure 18 and Figure 19, it is clearly visible that a $20 \mathrm{~Hz}$ peak appears in the LES signal spectra when the velocity and pressure probe is located within the cylinder wake (lower plots). Such peak is accurately reproduced by both PODI and DMD results.

The top plots in both Figures 18 and 19 show that all the reduced models considered allow for a sufficiently good reconstruction of the solution spectra for a point outside of the wake. In fact, all the yellow dashed curves appear very close to the black continuous one representing the LES 

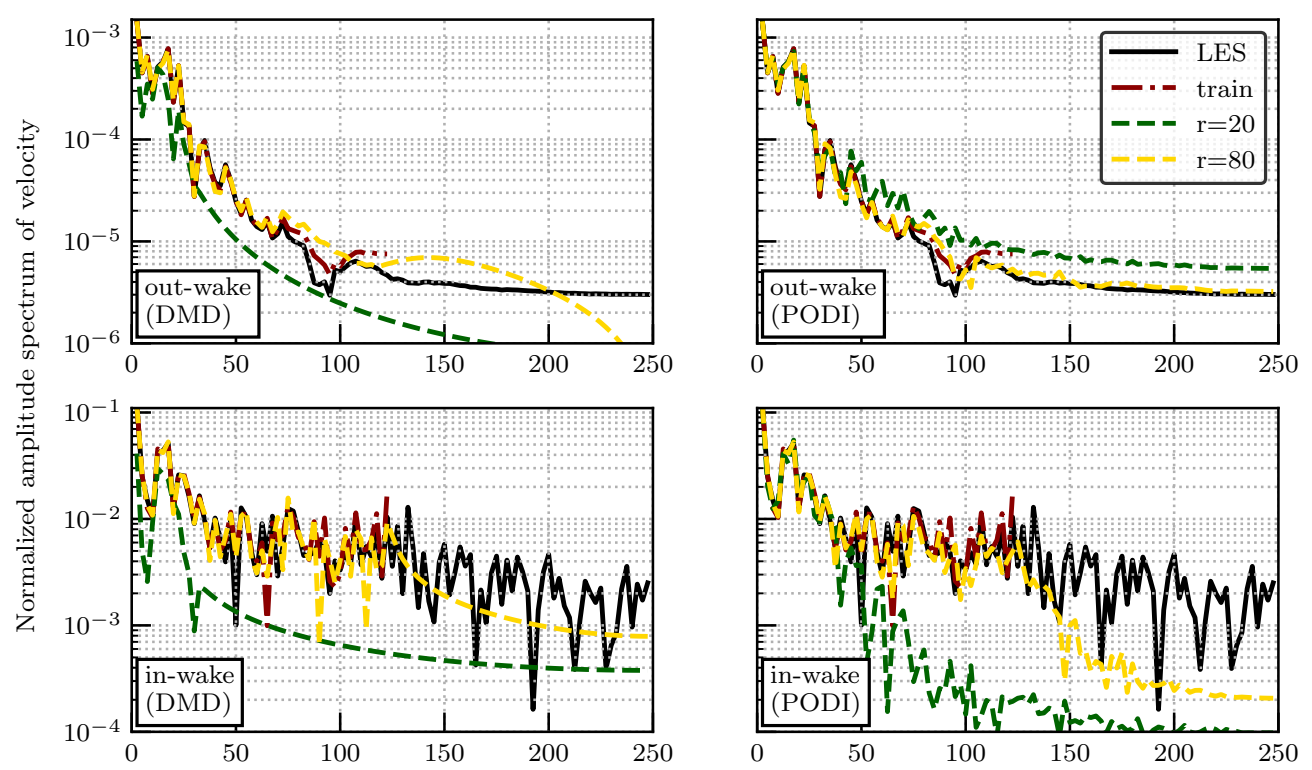

Frequency $[\mathrm{Hz}]$

Figure 18: Normalized amplitude spectrum of the streamwise velocity component, computed as (|fft $\left.(U-\bar{U}) \mid / N_{\text {freq }}\right)$ with $\bar{U}$ denoting mean velocity, in a fixed point located outside the wake (top) and inside the wake (bottom) for DMD (left) and PODI (right) at different truncation ranks (r). Black continuous and red dash-dotted lines refer to LES results of the full dataset and train dataset, respectively. Green and yellow dashed lines mark ROM surrogates employing 20 and 80 modes, respectively.

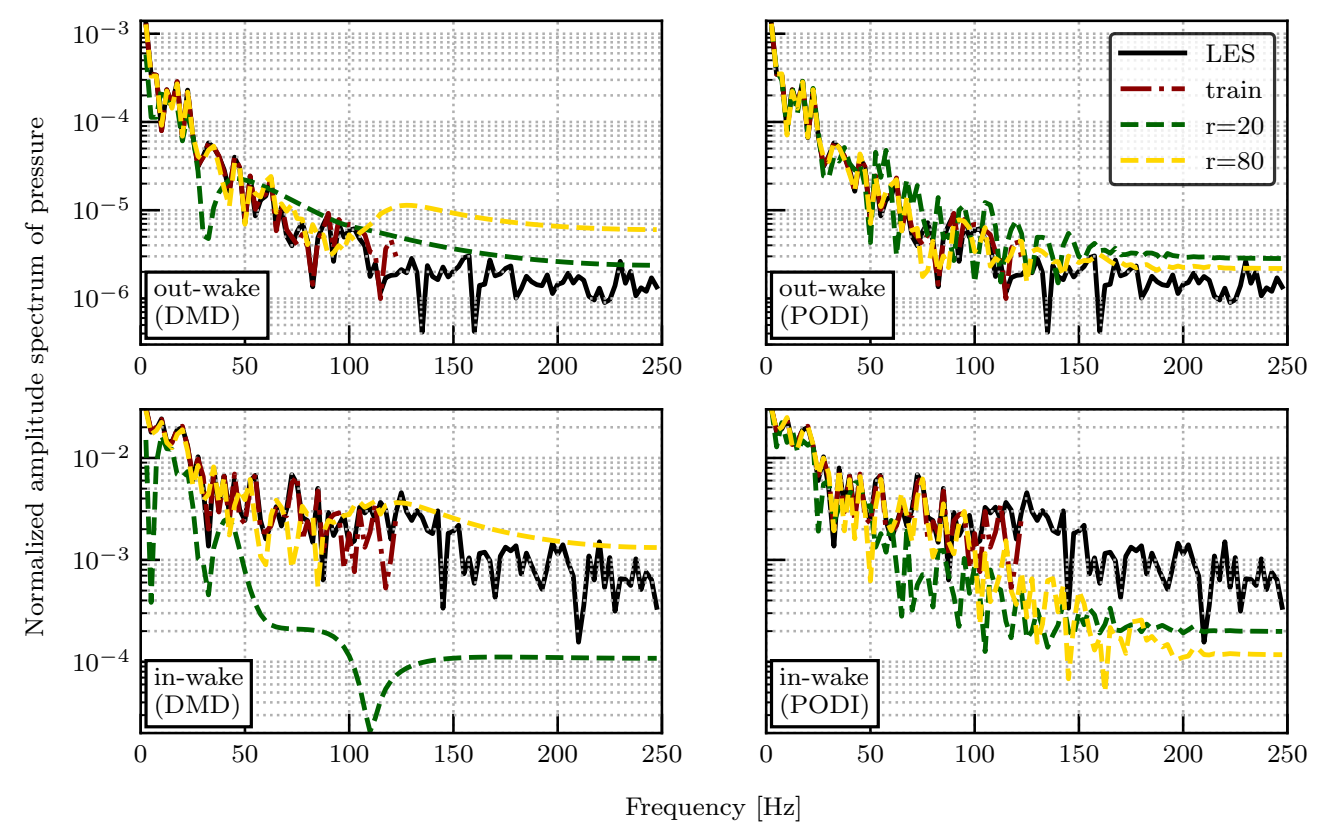

Figure 19: Normalized amplitude spectrum of the pressure signal, computed as $\left(|\operatorname{fft}(p-\bar{p})| / N_{\text {freq }}\right)$ with $\bar{p}$ denoting the mean pressure, in a fixed point located outside the wake (top) and inside the wake (bottom) for DMD (left) and PODI (right) at different truncation ranks (r). Color arrangements are same as in Figure 18. 
solution spectrum. Thus, this confirms that considering 80 modes or more leads to a spectrum that is indistinguishable from the original, especially for the PODI results in which a relative superiority is again noted in comparison with the spectra from DMD models. Yet, the higher frequency turbulent structures occur in the wake. In such region, as suggested by the bottom plots in Figures 18 and 19 , the frequency content of the streamwise velocity and the pressure solutions is definitely richer, and the accuracy of the reduced PODI and DMD solutions is clearly lower when higher frequencies are considered. Here, for velocity signals, the plot suggests that considering 80 modes both PODI and DMD algorithms lead to good quantitative spectral reconstructions of the velocity signal for frequencies up to approximately $110 \mathrm{~Hz}$. Interestingly, being based on the train signal yellow dashed line, in this case the PODI and DMD algorithms seem able to improve the behavior of the signal interpolated at half the sampling frequency, and somewhat extend its accuracy at frequencies very close to the Nyquist one.

It is worth commenting on the ROM sensitivity against grid resolution. When a coarser grid is utilized, larger length scales are modeled. As it is expected to note deviations in the corresponding FOM performance with respect to DNS data, it is also doubtful to expect recovering these missing details via a ROM. Furthermore, ROM predictions closely correlate with the energetic content and the induced frequencies from the employed dataset and respective modes. This implies that, while shifting from LES towards RANS grid resolutions, the underlying field dynamics are expected to be sufficiently recovered with less snapshots and less modes for the ROM.

\subsection{Acoustic analysis}

The acoustic analysis carried out in this work is aimed at comparing FWH signal obtained using the full order model data and the corresponding signal obtained with both the PODI and the DMD reduced order models. By a practical standpoint, the FWH post processing described in subsection 2.2 is applied to the pressure and velocity fields obtained from the LES full order simulation, and from both the PODI and DMD reduced models. The FWH integrals are here computed considering two microphones located at $\mathbf{x}_{\text {mic } \mathrm{A}}=\{0,2 D, 0\}$ (microphone $\mathrm{A}$ ) and $\mathbf{x}_{\text {mic } \mathrm{B}}=\{2 D, 2 D, 0\}$ (microphone B). As mentioned, the sphere is centered at the origin $\mathbf{O}=\{0,0,0\}$ and has diameter $D$. The acoustic pressure time-history is converted to sound Spectrum Level $\mathrm{SpL}=20 \log \left(p / p_{\text {ref }}\right)$, considering $p_{\text {ref }}=1 \mu \mathrm{Pa}$.

We will first focus on the results of the PODI model. The corresponding SpL from both microphones is reported in Figures 20 and 21, right panels. In the figures, the different FWH signal curves denote the different number of modes employed in the PODI reconstruction. Also, as seen in previous works where the FWH formulation is used, it is convenient to separate the linear contribution to the acoustic pressure, obtained from the surface integrals in Equation 3, from the nonlinear part obtained from the volume integrals in Equation 4 Such contributions are referred to as dipole and quadrupole terms, respectively. This distinction is particularly relevant, as the former contribution only requires the knowledge of the pressure field on the body surface, while the latter component takes into consideration the evolution of the pressure and velocity field in the wake region. More specifically, we emphasize that to obtain an adequate reconstruction of the non-linear acoustic signal, an accurate reconstruction of the vorticity field is necessary.

In general, the PODI results seem satisfactory especially for what concerns utilizing 80 modes. Figures 20 and 21 (right top plots) show in fact good agreement between the linear acoustic pressure contribution based on the LES pressure field and the corresponding linear contribution computed with PODI employing 80 modes (yellow lines in the plots). As the plots suggest, this trend is appreciable both at microphones A and B. As for the nonlinear contribution, Figures 20 and 21 (right bottom panels) also show a satisfactory agreement between the acoustic pressure signals obtained from the LES flow fields and those computed based on PODI. In this case, in the plots referring to both microphones, the blue and yellow lines (40 and 80 modes respectively) on the right bottom panels are sufficiently close to their reference FOM counterpart, represented by the continuous black line.

At a closer look, close agreement between PODI and FOM acoustic pressures, both for the linear and non-linear contributions, can be established at least up to frequencies in the range 80$100 \mathrm{~Hz}$, which may be considered the meaningful frequency interval related to the considered test case. In fact, we point out that, since the reference pressure is set to $1 \mu \mathrm{Pa}$, the ambient noise may be considered in the range of 60-100 dB. At higher frequencies though, we note that some spurious 


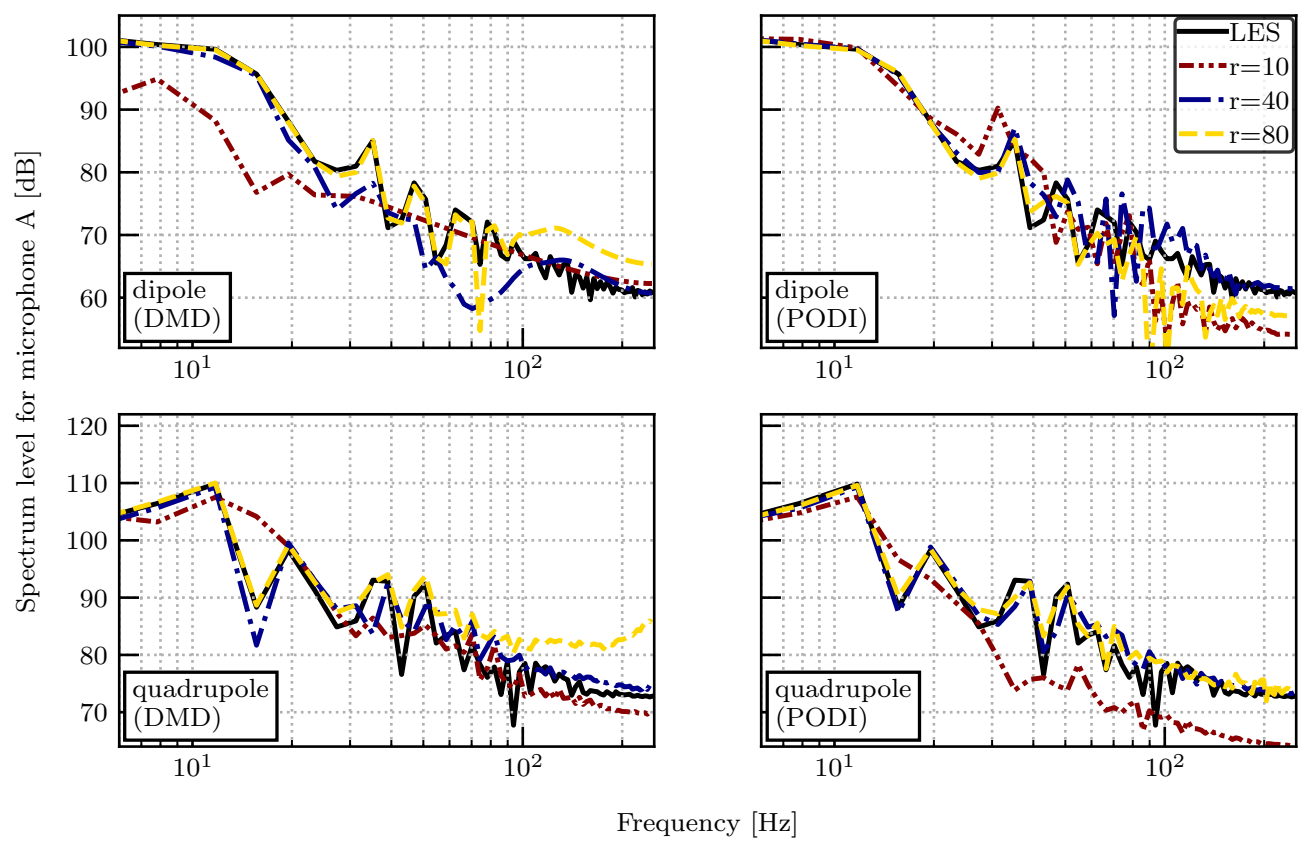

Figure 20: Linear dipole (top) and nonlinear quadrupole (bottom) terms of FWH equation evaluated from LES data and compared to corresponding DMD (left) and PODI (right) reduced models at different truncation ranks (r). Microphone A.

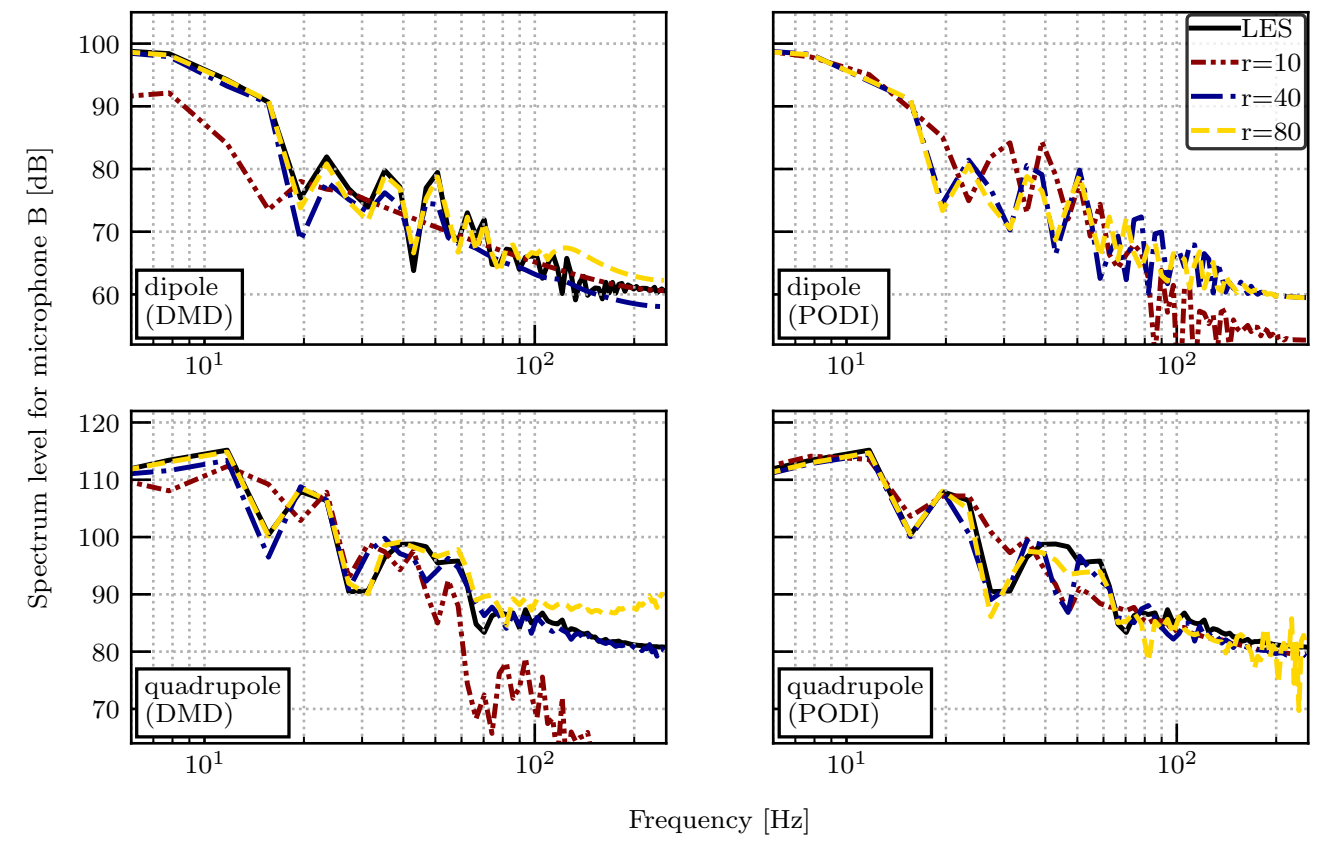

Figure 21: Linear dipole (top) and nonlinear quadrupole (bottom) terms of FWH equation evaluated from LES data and compared to corresponding DMD (left) and PODI (right) reduced models at different truncation ranks (r). Microphone B. 
oscillations appear in the PODI results, especially in the nonlinear noise terms corresponding to microphone B (Figure 21), at which the value of the integrals in the FWH formulation are strongly conditioned by the presence of the wake. The observed spurious oscillations could be due to an imperfect reconstruction of the vorticity field, although it must be emphasized that the sampling frequency of the original dataset is not very high, $f_{s}=500 \mathrm{~Hz}$, thereby for the train dataset it becomes $f_{s}=250 \mathrm{~Hz}$ for such an alternative arrangement. Therefore, it is reasonable to obtain less accurate results at frequencies higher than $125 \mathrm{~Hz}$. Further work will be devoted to investigating whether longer temporal records or higher sampling frequencies will eliminate or mitigate the problem.

However, considering the 80 modes PODI reconstruction, the maximum error is of the order of $5 \mathrm{~dB}$, observed at microphone $\mathrm{B}$, for the non linear terms (Figure 21 bottom right panel). In addition, the main peaks observed at very low frequencies, up to $20 \mathrm{~Hz}$, are well captured by the PODI signal. In such case, the agreement is also verified for the 40 modes signals. As these low frequency peaks describe the main and most energetic features of the acoustic signal, this indicates that a moderate amount of modes might still be a good compromise when the acoustic analysis is only aiming at a general characterization of the principal noise features. Finally, we must point out that the errors observed in the non-linear noise contributions are not in general higher than those introduced in the linear contributions, as might be expected given the interpolation procedure involved in PODI. In some occurrence (Figure 20 top right plot), the linear contribution errors are even quite surprisingly higher than their nonlinear counterparts. This might be related to the fact that the linear noise contributions are only based on pressure evaluations on the body surface, while the non-linear contributions are based on volume integrals. The POD procedure used selects the modal shapes so as to minimize the error in a norm based on the whole volumetric solution, rather than only on a surface restriction. For such reason the modal shape selected might result in non optimal results in the computation of surface integrals. Possible gains might then be obtained, if needed, adding a separate PODI only built on the body surface degrees of freedom, which would result in a modest increase of the computational cost.

As regards the acoustic signals provided by the DMD method, a good agreement is observed with respect to the FOM spectrum, up to about $80 \mathrm{~Hz}$, for both microphones, and both linear and non-linear terms, see Figures 20 and 21 (left panels). The most accurate signals are obtained using 40 and 80 modes, while for the signal related to 10 modes we observe, as expected, a considerable discrepancy. In general, the reconstruction of the vorticity field, obtained by both POD and DMD, is found to provide an adequate input field for the acoustic model. Particularly, the volume integral of the FWH equation involves both the velocity and the pressure field in the wake region. Having observed a good match of the ROM signals with respect to the FOM reference signals, we may conclude that the entire spectrum associated with the vortex wake has been adequately reconstructed.

\section{Conclusions and perspectives}

This work discussed the application of data-driven dimensionality reduction algorithms on a hydroacoustic dataset which was numerically measured using Large Eddy Simulation (LES) fluid dynamic turbulence model and the Ffowcs Williams and Hawkings (FWH) acoustic analogy. An extensive set of data was presented to fully characterize the ability of both Dynamic Mode Decomposition (DMD) and Proper Orthogonal Decomposition (POD) to reconstruct the flow fields, based on their spectral and energetic contents, with all spatial and temporal frequencies needed to support accurate noise predictions.

First, Singular Value Decomposition (SVD) analysis did not indicate the presence of significant constraints on the modal truncation rank for such flows. In fact, no significant singular value energy gaps were observed. SVD was then used to extract DMD and POD modes with associated coefficients, and to employ them for flow fields reconstruction. In general, both DMD and POD algorithms showed efficient reconstruction accuracy. Spatial and temporal error analyses indicated relatively lower error magnitudes in POD-based reconstructed fields. On the other hand, statistical analysis and vortical structures identification methods demonstrated the ability of DMD to capture finest wake scales by employing higher modes, compared with POD.

Second, two data-driven reduced models based on DMD mid cast and on POD with interpolation (PODI) were created utilizing half the LES original dataset. Both DMD and POD based reduced models showed good efficiency and accurate flow reconstruction. In addition, the spectral 

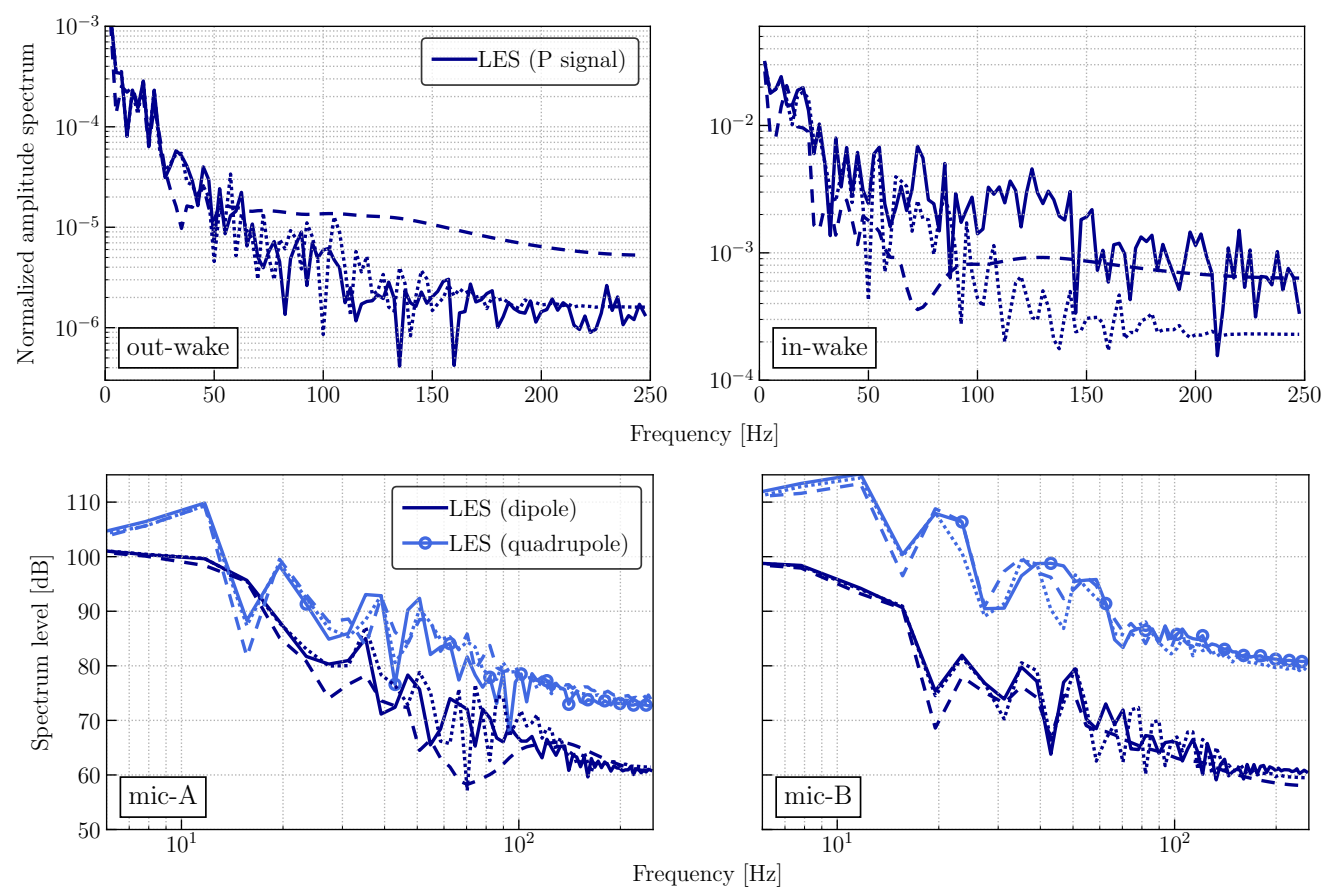

Figure 22: Top: FFT spectrum of the pressure signal at points located out of wake (left) and inside the wake (right). Bottom: dipole and quadrupole acoustic noise spectrum measured at mic-A (left) and mic-B (right) locations. PODI spectra (....) demonstrate better agreement with LES data (-) compared with DMD (- - ) ones. Both PODI and DMD results in the graphs have been obtained employing $r=40$ modes.

analysis of the reduced flow solutions at selected points inside and outside vortical wake regions indicated that both models were able to recover most of the model flow frequencies in the ranges of interest for the acoustic analysis. In particular, PODI showed notable capability to capture additional frequencies which were present in the original dataset, but not in the subset employed to train the model. As a consequence, both data-driven reduced models developed proved efficient and sufficiently accurate in predicting acoustic noises.

Given the results obtained, introducing bluff body geometrical parameterization as additional dimension for the PODI analysis is an interesting possibility for future works. A further future perspective which is currently being explored, is represented by the development of a reduced model employing the POD modal decomposition for the Galerkin projection of the continuity and momentum equations for the fluid dynamic variables. Other studies could be devoted to better understand how the choice of the interpolator for PODI affects the results.

\section{Appendix}

\section{A ROMs cross-comparison}

For additional clarity, the spectral and acoustic analyses discussed in the previous sections, cf.Figures 18 to 21, are here reported in single graphs directly comparing both DMD and PODI ROMs results against LES data. In particular, we are considering both DMD and PODI ROMs truncated at $r=40$. The aim is to provide an easier comparison of the ROMs performance. Figure 22 depicts FFT spectrum of the pressure signal (inside and outside the wake, top plots) and the dipole and quadrupole acoustic noises (microphones $\mathrm{A}$ and $\mathrm{B}$, bottom plots). In all plots DMD (- - ) and PODI (....) results are reported alongside LES (-) results. The plots clearly indicate that, given equal modal truncation level, PODI models appear to accurately recover a wider range of frequencies compared to DMD models. 


\section{Acknowledgements}

This work was partially supported by the project PRELICA, "Advanced methodologies for hydroacoustic design of naval propulsion", supported by Regione FVG, POR-FESR 2014-2020, Piano Operativo Regionale Fondo Europeo per lo Sviluppo Regionale, and partially funded by European Union Funding for Research and Innovation - Horizon 2020 Program - in the framework of European Research Council Executive Agency: H2020 ERC CoG 2015 AROMA-CFD project 681447 "Advanced Reduced Order Methods with Applications in Computational Fluid Dynamics" P.I. Gianluigi Rozza. We gratefully thank professor Vincenzo Armenio, University of Trieste, for the general supervision, his precious comments and suggestions, during the development of the presented work.

\section{References}

[1] D.-G. Baek, H.-S. Yoon, J.-H. Jung, K.-S. Kim, and B.-G. Paik. Effects of the advance ratio on the evolution of a propeller wake. Computers \& Fluids, 118:32-43, Sept. 2015.

[2] E. Balaras, S. Schroeder, and A. Posa. Large-Eddy Simulations of Submarine Propellers. Journal of Ship Research, 59(4):227-237, Dec. 2015.

[3] G. Berkooz, P. Holmes, and J. L. Lumley. The Proper Orthogonal Decomposition in the Analysis of Turbulent Flows. Annual Review of Fluid Mechanics, 25(1):539-575, Jan. 1993.

[4] D. A. Bistrian and I. M. Navon. An improved algorithm for the shallow water equations model reduction: Dynamic Mode Decomposition vs POD. International Journal for Numerical Methods in Fluids, 78(9):552-580, Apr. 2015.

[5] D. A. Bistrian and I. M. Navon. Randomized dynamic mode decomposition for nonintrusive reduced order modelling. International Journal for Numerical Methods in Engineering, 112(1):3-25, 2017.

[6] D. A. Bistrian and I. M. Navon. Efficiency of randomised dynamic mode decomposition for reduced order modelling. International Journal of Computational Fluid Dynamics, 32(23):88-103, 2018.

[7] E. Bouhoubeiny and P. Druault. Note on the POD-based time interpolation from successive PIV images. Comptes Rendus Mécanique, 337(11-12):776-780, Nov. 2009.

[8] K. S. Brentner and F. Farassat. Modeling aerodynamically generated sound of helicopter rotors. Progress in Aerospace Sciences, 39(2-3):83-120, Feb. 2003.

[9] A. Broatch, J. García-Tíscar, F. Roig, and S. Sharma. Dynamic mode decomposition of the acoustic field in radial compressors. Aerospace Science and Technology, 90:388-400, 2019.

[10] S. L. Brunton and J. N. Kutz. Data-Driven Science and Engineering: Machine Learning, Dynamical Systems, and Control. Cambridge University Press, 2019.

[11] T. Bui-Thanh, M. Damodaran, and K. Willcox. Proper Orthogonal Decomposition Extensions for Parametric Applications in Compressible Aerodynamics. In 21st AIAA Applied Aerodynamics Conference, page 4213, 2003.

[12] T. Bui-Thanh, M. Damodaran, and K. E. Willcox. Aerodynamic Data Reconstruction and Inverse Design Using Proper Orthogonal Decomposition. AIAA journal, 42(8):1505-1516, 2004.

[13] E. F. Campana, D. Peri, Y. Tahara, and F. Stern. Shape optimization in ship hydrodynamics using computational fluid dynamics. Computer Methods in Applied Mechanics and Engineering, 196(1-3):634-651, Dec. 2006.

[14] M. Cianferra, V. Armenio, and S. Ianniello. Hydroacoustic noise from different geometries. International Journal Heat Fluid Flow, 70:348-362, 2018. 
[15] M. Cianferra, S. Ianniello, and V. Armenio. Assessment of methodologies for the solution of the Ffowcs Williams and Hawkings equation using LES of incompressible single-phase flow around a finite-size square cylinder. Journal of Sound and Vibration, 453:1-24, 2019.

[16] M. Cianferra, A. Petronio, and V. Armenio. Hydrodynamic Noise from a Propeller in Open Sea Condition. In Technology and Science for the Ships of the Future: Proceedings of NAV 2018: 19th International Conference on Ship \& Maritime Research, pages 149-156. IOS Press, 2018.

[17] M. Cianferra, A. Petronio, and V. Armenio. Non-linear noise from a ship propeller in open sea condition. Ocean Engineering, 191:106474, Nov. 2019.

[18] C. Cintolesi, A. Petronio, and V. Armenio. Large eddy simulation of turbulent buoyant flow in a confined cavity with conjugate heat transfer. Phys fluids, 27, 2015.

[19] M. F. de Pando, P. J. Schmid, and D. Sipp. A global analysis of tonal noise in flows around aerofoils. Journal of Fluid Mechanics, 754:5-38, July 2014.

[20] N. Demo, M. Tezzele, G. Gustin, G. Lavini, and G. Rozza. Shape Optimization by Means of Proper Orthogonal Decomposition and Dynamic Mode Decomposition. In Technology and Science for the Ships of the Future: Proceedings of NAV 2018: 19th International Conference on Ship 8 Maritime Research, pages 212-219. IOS Press, 2018.

[21] N. Demo, M. Tezzele, A. Mola, and G. Rozza. An Efficient Shape Parametrisation by FreeForm Deformation Enhanced by Active Subspace for Hull Hydrodynamic Ship Design Problems in Open Source Environment. In Proceedings of ISOPE 2018: The 28th International Ocean and Polar Engineering Conference, volume 3, pages 565-572, 2018.

[22] N. Demo, M. Tezzele, A. Mola, and G. Rozza. A complete data-driven framework for the efficient solution of parametric shape design and optimisation in naval engineering problems. In R. Bensow and J. Ringsberg, editors, Proceedings of MARINE 2019: VIII International Conference on Computational Methods in Marine Engineering, pages 111-121, 2019.

[23] N. Demo, M. Tezzele, and G. Rozza. EZyRB: Easy Reduced Basis method. The Journal of Open Source Software, 3(24):661, 2018.

[24] N. Demo, M. Tezzele, and G. Rozza. PyDMD: Python Dynamic Mode Decomposition. The Journal of Open Source Software, 3(22):530, 2018.

[25] N. Demo, M. Tezzele, and G. Rozza. A non-intrusive approach for reconstruction of POD modal coefficients through active subspaces. Comptes Rendus Mécanique de l'Académie des Sciences, DataBEST 2019 Special Issue, 347(11):873-881, November 2019.

[26] V. Dolci and R. Arina. Proper Orthogonal Decomposition as Surrogate Model for Aerodynamic Optimization. International Journal of Aerospace Engineering, 2016.

[27] P. Druault and C. Chaillou. Use of Proper Orthogonal Decomposition for reconstructing the 3D in-cylinder mean-flow field from PIV data. Comptes Rendus Mécanique, 335(1):42-47, Jan. 2007.

[28] N. B. Erichson, S. L. Brunton, and J. N. Kutz. Compressed dynamic mode decomposition for background modeling. Journal of Real-Time Image Processing, 16(5):1479-1492, Oct 2019.

[29] J. E. Ffowcs Williams and D. L. Hawkings. Sound generation by turbulence and surfaces in arbitrary motion. Philosophical Transaction of Royal Society, 264:321-342, 1969.

[30] M. Fossati and W. G. Habashi. Multiparameter Analysis of Aero-Icing Problems Using Proper Orthogonal Decomposition and Multidimensional Interpolation. AIAA Journal, 51(4):946-960, Apr. 2013.

[31] T. Franz, R. Zimmermann, S. Görtz, and N. Karcher. Interpolation-based reduced-order modelling for steady transonic flows via manifold learning. International Journal of Computational Fluid Dynamics, 28(3-4):106-121, Mar. 2014. 
[32] M. Gadalla, M. Tezzele, A. Mola, and G. Rozza. BladeX: Python Blade Morphing. The Journal of Open Source Software, 4(34):1203, 2019.

[33] F. Garotta, N. Demo, M. Tezzele, M. Carraturo, A. Reali, and G. Rozza. Reduced Order Isogeometric Analysis Approach for PDEs in Parametrized Domains. In M. D'Elia, M. Gunzburger, and G. Rozza, editors, Quantification of Uncertainty: Improving Efficiency and Technology: QUIET selected contributions, volume 137 of Lecture Notes in Computational Science and Engineering, pages 153-170. Springer International Publishing, Cham, 2020.

[34] S. Georgaka, G. Stabile, K. Star, G. Rozza, and M. J. Bluck. A hybrid reduced order method for modelling turbulent heat transfer problems. Computers \& Fluids, 208:104615, 2020.

[35] S. A. Glegg and W. J. Devenport. Proper orthogonal decomposition of turbulent flows for aeroacoustic and hydroacoustic applications. Journal of Sound and Vibration, 239(4):767$784,2001$.

[36] X. Gloerfelt, F. Pérot, C. Bailly, and D. Juvé. Flow-induced cylinder noise formulated as a diffraction problem for low Mach numbers. Journal of Sound and Vibration, 287(1-2):129151, Oct. 2005.

[37] Y. L. Guennec, J.-P. Brunet, F.-Z. Daim, M. Chau, and Y. Tourbier. A parametric and non-intrusive reduced order model of car crash simulation. Computer Methods in Applied Mechanics and Engineering, 338:186-207, Aug. 2018.

[38] G. Haller. An objective definition of a vortex. Journal of Fluid Mechanics, 525:1-26, Feb. 2005.

[39] J. S. Hesthaven, G. Rozza, B. Stamm, et al. Certified Reduced Basis Methods for Parametrized Partial Differential Equations. Springer, 2016.

[40] S. Hijazi, G. Stabile, A. Mola, and G. Rozza. Data-Driven POD-Galerkin reduced order model for turbulent flows. Journal of Computational Physics, 416:109513, 2020.

[41] S. Ianniello, R. Muscari, and A. D. Mascio. Ship underwater noise assessment by the acoustic analogy. Part I: nonlinear analysis of a marine propeller in a uniform flow. Journal of Marine Science and Technology, 18(4):547-570, July 2013.

[42] S. Ianniello, R. Muscari, and A. D. Mascio. Ship underwater noise assessment by the acoustic analogy. Part II: hydroacoustic analysis of a ship scaled model. J Mar Sci Technol, 18(4):547$570,2013$.

[43] E. Iuliano and D. Quagliarella. Proper Orthogonal Decomposition, surrogate modelling and evolutionary optimization in aerodynamic design. Computers 83 Fluids, 84:327-350, Sept. 2013.

[44] G. Jourdain, L.-E. Eriksson, S. H. Kim, and C. H. Sohn. Application of dynamic mode decomposition to acoustic-modes identification and damping in a 3-dimensional chamber with baffled injectors. Journal of Sound and Vibration, 332(18):4308-4323, Sept. 2013.

[45] M. R. Jovanović, P. J. Schmid, and J. W. Nichols. Sparsity-promoting dynamic mode decomposition. Physics of Fluids, 26(2):024103, Feb 2014.

[46] S. Karri, J. Charonko, and P. P. Vlachos. Robust wall gradient estimation using radial basis functions and proper orthogonal decomposition (POD) for particle image velocimetry (PIV) measured fields. Measurement Science and Technology, 20(4):045401, Feb. 2009.

[47] J. E. Kerwin. Marine Propellers. Annual Review of Fluid Mechanics, 18(1):367-403, Jan. 1986.

[48] P. Kumar and K. Mahesh. Large eddy simulation of propeller wake instabilities. Journal of Fluid Mechanics, 814:361-396, Feb. 2017.

[49] K. Kunisch and S. Volkwein. Proper orthogonal decomposition for optimality systems. ESAIM: Mathematical Modelling and Numerical Analysis, 42(1):1-23, Jan. 2008. 
[50] J. N. Kutz, S. L. Brunton, B. W. Brunton, and J. L. Proctor. Dynamic Mode Decomposition: Data-Driven Modeling of Complex Systems, volume 149. SIAM, 2016.

[51] J. N. Kutz, X. Fu, and S. L. Brunton. Multiresolution Dynamic Mode Decomposition. SIAM Journal on Applied Dynamical Systems, 15(2):713-735, 2016.

[52] S. Le Clainche and J. M. Vega. Higher Order Dynamic Mode Decomposition. SIAM Journal on Applied Dynamical Systems, 16(2):882-925, 2017.

[53] L. S. Lorente, J. M. Vega, and A. Velazquez. Generation of Aerodynamics Databases Using High-Order Singular Value Decomposition. Journal of Aircraft, 45(5):1779-1788, Sept. 2008.

[54] H. V. Ly and H. T. Tran. Modeling and control of physical processes using proper orthogonal decomposition. Mathematical and Computer Modelling, 33(1-3):223-236, Jan. 2001.

[55] M. Mancinelli, T. Pagliaroli, R. Camussi, and T. Castelain. On the hydrodynamic and acoustic nature of pressure proper orthogonal decomposition modes in the near field of a compressible jet. Journal of Fluid Mechanics, 836:998-1008, Dec. 2017.

[56] I. Markovsky. Low-Rank Approximation. Communications and Control Engineering. Springer International Publishing, 2 edition, 2019.

[57] A. D. Mascio, R. Muscari, and G. Dubbioso. On the wake dynamics of a propeller operating in drift. Journal of Fluid Mechanics, 754:263-307, July 2014.

[58] C. Meneveau, T. Lund, and W. Cabot. A Lagrangian dynamic subgrid-scale model of turbulence. J Fluid Mech, 319:353-385, 1996.

[59] K. E. Meyer, J. M. Pedersen, and O. Özcan. A turbulent jet in crossflow analysed with proper orthogonal decomposition. Journal of Fluid Mechanics, 583:199-227, July 2007.

[60] M. J. Mifsud, D. G. MacManus, and S. Shaw. A variable-fidelity aerodynamic model using proper orthogonal decomposition. International Journal for Numerical Methods in Fluids, 82(10):646-663, Apr. 2016.

[61] M. J. Mifsud, S. T. Shaw, and D. G. MacManus. A high-fidelity low-cost aerodynamic model using proper orthogonal decomposition. International Journal for Numerical Methods in Fluids, pages n/a-n/a, 2009.

[62] A. Mola, M. Tezzele, M. Gadalla, F. Valdenazzi, D. Grassi, R. Padovan, and G. Rozza. Efficient Reduction in Shape Parameter Space Dimension for Ship Propeller Blade Design. In R. Bensow and J. Ringsberg, editors, Proceedings of MARINE 2019: VIII International Conference on Computational Methods in Marine Engineering, pages 201-212, 2019.

[63] T. W. Muld, G. Efraimsson, and D. S. Henningson. Flow structures around a high-speed train extracted using Proper Orthogonal Decomposition and Dynamic Mode Decomposition. Computers \& Fluids, 57:87-97, 2012.

[64] A. Najafi-Yazdi, G.A.Bres, and L. Mongeau. An acoustic analogy formulation for moving sources in uniformly moving media. Proc R Soc Lond, A467:144-165, 2011.

[65] Z. Nitzkorski and K. Mahesh. A dynamic end cap technique for sound computation using the Ffowcs Williams and Hawkings equations. Physics of Fluids, 26(11):115101, Nov. 2014.

[66] U. Piomelli, L. S. Craig, and S. Sarkar. On the computation of sound by large-eddy simulations. Journal of Engineering and Mathematics, 32:217-236, 1997.

[67] A. Posa, R. Broglia, M. Felli, M. Falchi, and E. Balaras. Characterization of the wake of a submarine propeller via Large-Eddy simulation. Computer and Fluids, 184:138-152, 2019.

[68] J. L. Proctor, S. L. Brunton, and J. N. Kutz. Dynamic Mode Decomposition with Control. SIAM Journal on Applied Dynamical Systems, 15(1):142-161, 2016.

[69] A. Quarteroni, A. Manzoni, and F. Negri. Reduced Basis Methods for Partial Differential Equations: An Introduction, volume 92. Springer, 2015. 
[70] M. Ripepi, M. Verveld, N. Karcher, T. Franz, M. Abu-Zurayk, S. Görtz, and T. Kier. Reduced-order models for aerodynamic applications, loads and MDO. CEAS Aeronautical Journal, 9(1):171-193, 2018.

[71] C. W. Rowley, T. Colonius, and R. M. Murray. Model reduction for compressible flows using POD and Galerkin projection. Physica D: Nonlinear Phenomena, 189(1-2):115-129, Feb. 2004.

[72] C. W. Rowley, I. Mezić, S. Bagheri, P. Schlatter, and D. S. Henningson. Spectral analysis of nonlinear flows. Journal of fluid mechanics, 641:115-127, 2009.

[73] G. Rozza, M. W. Hess, G. Stabile, M. Tezzele, and F. Ballarin. Basic Ideas and Tools for Projection-Based Model Reduction of Parametric Partial Differential Equations . In P. Benner, S. Grivet-Talocia, A. Quarteroni, G. Rozza, W. H. A. Schilders, and L. M. Silveira, editors, Handbook on Model Order Reduction, volume 2, chapter 1. De Gruyter, In Press, 2020.

[74] G. Rozza, M. H. Malik, N. Demo, M. Tezzele, M. Girfoglio, G. Stabile, and A. Mola. Advances in Reduced Order Methods for Parametric Industrial Problems in Computational Fluid Dynamics. In R. Owen, R. de Borst, J. Reese, and P. Chris, editors, ECCOMAS ECFD 7 - Proceedings of 6th European Conference on Computational Mechanics (ECCM 6) and 7th European Conference on Computational Fluid Dynamics (ECFD 7), pages 59-76, Glasgow, UK, 2018.

[75] S. H. Rudy, J. N. Kutz, and S. L. Brunton. Deep learning of dynamics and signal-noise decomposition with time-stepping constraints. J. Comput. Physics, 396:483-506, 2018.

[76] H. Sakamoto and H. Haniu. A Study on Vortex Shedding From Spheres in a Uniform Flow. Journal of Fluids Engineering, 112(4):386-392, 121990.

[77] F. Salmoiraghi, F. Ballarin, G. Corsi, A. Mola, M. Tezzele, and G. Rozza. Advances in geometrical parametrization and reduced order models and methods for computational fluid dynamics problems in applied sciences and engineering: overview and perspectives. ECCOMAS Congress 2016 - Proceedings of the 7th European Congress on Computational Methods in Applied Sciences and Engineering, 1:1013-1031, 2016.

[78] F. Salmoiraghi, A. Scardigli, H. Telib, and G. Rozza. Free-form deformation, mesh morphing and reduced-order methods: enablers for efficient aerodynamic shape optimisation. International Journal of Computational Fluid Dynamics, 32(4-5):233-247, 2018.

[79] R. D. Sandberg and N. D. Sandham. Direct numerical simulation of turbulent flow past a trailing edge and the associated noise generation. Journal of Fluid Mechanics, 596:353-385, Jan. 2008.

[80] P. J. Schmid. Dynamic mode decomposition of numerical and experimental data. Journal of fluid mechanics, 656:5-28, 2010.

[81] P. J. Schmid. Application of the dynamic mode decomposition to experimental data. Experiments in fluids, 50(4):1123-1130, 2011.

[82] P. J. Schmid, L. Li, M. P. Juniper, and O. Pust. Applications of the dynamic mode decomposition. Theoretical and Computational Fluid Dynamics, 25(1-4):249-259, 2011.

[83] A. Seena and H. J. Sung. Dynamic mode decomposition of turbulent cavity flows for selfsustained oscillations. International Journal of Heat and Fluid Flow, 32(6):1098-1110, Dec. 2011.

[84] J. H. Seo, Y. J. Moon, and B. R. Shin. Prediction of cavitating flow noise by direct numerical simulation. Journal of Computational Physics, 227(13):6511-6531, June 2008.

[85] H. Seol, B. Jung, J.-C. Suh, and S. Lee. Prediction of non-cavitating underwater propeller noise. Journal of Sound and Vibration, 257(1):131-156, Oct. 2002.

[86] C. Seror, P. Sagaut, C. Bailly, and D. Juvé. On the radiated noise computed by large-eddy simulation. Physics of Fluids, 13(2):476-487, Feb. 2001. 
[87] W. Shen, T. K. Patel, and S. A. Miller. Extraction of Large-Scale Coherent Structures from Large Eddy Simulation of Supersonic Jets for Shock-Associated Noise Prediction. In AIAA Scitech 2020 Forum. American Institute of Aeronautics and Astronautics, Jan. 2020.

[88] L. Sirovich. Turbulence and the dynamics of coherent structures. II. symmetries and transformations. Quarterly of Applied Mathematics, 45(3):573-582, Oct. 1987.

[89] G. Stabile, F. Ballarin, G. Zuccarino, and G. Rozza. A reduced order variational multiscale approach for turbulent flows. Advances in Computational Mathematics, 45:2349-2368, 2019.

[90] G. Stabile, S. Hijazi, A. Mola, S. Lorenzi, and G. Rozza. POD-Galerkin reduced order methods for CFD using Finite Volume Discretisation: vortex shedding around a circular cylinder. Communications in Applied and Industrial Mathematics, 8(1):210-236, 2017.

[91] G. Stabile and G. Rozza. Finite volume POD-Galerkin stabilised reduced order methods for the parametrised incompressible Navier-Stokes equations. Computers \& Fluids, 173:273-284, sep 2018.

[92] P. Stegeman, A. Ooi, and J. Soria. Proper Orthogonal Decomposition and Dynamic Mode Decomposition of Under-Expanded Free-Jets with Varying Nozzle Pressure Ratios. In Instability and Control of Massively Separated Flows, pages 85-90. Springer, 2015.

[93] M. Tezzele, N. Demo, M. Gadalla, A. Mola, and G. Rozza. Model Order Reduction by Means of Active Subspaces and Dynamic Mode Decomposition for Parametric Hull Shape Design Hydrodynamics. In Technology and Science for the Ships of the Future: Proceedings of NAV 2018: 19th International Conference on Ship \&6 Maritime Research, pages 569-576. IOS Press, 2018.

[94] M. Tezzele, N. Demo, A. Mola, and G. Rozza. An integrated data-driven computational pipeline with model order reduction for industrial and applied mathematics. Special Volume ECMI, Springer, In Press, 2020.

[95] M. Tezzele, N. Demo, A. Mola, and G. Rozza. PyGeM: Python geometrical morphing. Software Impacts, page 100047, 2020.

[96] M. Tezzele, N. Demo, and G. Rozza. Shape optimization through proper orthogonal decomposition with interpolation and dynamic mode decomposition enhanced by active subspaces. In R. Bensow and J. Ringsberg, editors, Proceedings of MARINE 2019: VIII International Conference on Computational Methods in Marine Engineering, pages 122-133, 2019.

[97] M. Tezzele, N. Demo, G. Stabile, A. Mola, and G. Rozza. Enhancing CFD predictions in shape design problems by model and parameter space reduction. Advanced Modeling and Simulation in Engineering Sciences, 7(40), 2020.

[98] M. Tezzele, F. Salmoiraghi, A. Mola, and G. Rozza. Dimension reduction in heterogeneous parametric spaces with application to naval engineering shape design problems. Advanced Modeling and Simulation in Engineering Sciences, 5(1), 2018.

[99] F. Valdenazzi, F. Conti, S. Gaggero, D. Grassi, C. Vaccaro, and D. Villa. A practical tool for the hydro-acoustic optimization of naval propellers. In R. Bensow and J. Ringsberg, editors, Proceedings of MARINE 2019: VIII International Conference on Computational Methods in Marine Engineering, pages 296-308, 2019.

[100] H. G. Weller, G. Tabor, H. Jasak, and C. Fureby. A tensorial approach to computational continuum mechanics using object-oriented techniques. Computers in physics, 12(6):620-631, 1998.

[101] M. O. Williams, I. G. Kevrekidis, and C. W. Rowley. A Data-Driven Approximation of the Koopman Operator: Extending Dynamic Mode Decomposition. Journal of Nonlinear Science, 25(6):1307-1346, 2015.

[102] D. Xiao, F. Fang, C. Pain, and G. Hu. Non-intrusive reduced-order modelling of the NavierStokes equations based on RBF interpolation. International Journal for Numerical Methods in Fluids, 79(11):580-595, July 2015. 
[103] Q. Zhang, Y. Liu, and S. Wang. The identification of coherent structures using proper orthogonal decomposition and dynamic mode decomposition. Journal of Fluids and Structures, 49:53-72, 2014. 